\title{
Existence and features of similarity solutions for non-Boussinesq gravity currents
}

\author{
C. Ancey*, S. Cochard, M. Rentschler, S. Wiederseiner \\ École Polytechnique Fédérale de Lausanne, 1015 Lausanne, Switzerland
}

Received 22 June 2006; received in revised form 27 October 2006; accepted 1 November 2006

Available online 14 December 2006

Communicated by W. van Saarloos

\begin{abstract}
In this paper, the flow dynamics of gravity currents on a horizontal plane is investigated from a theoretical point of view by seeking similarity solutions. The current is generated by unleashing a varying volume of heavy fluid within an ambient fluid of much lower density. Unlike earlier investigators, we assume that the ambient fluid exerts no significant resisting action on the current, and therefore the flow depth is expected to drop to zero at the front in the absence of friction. In this context, the shallow-water equations are highly appropriate for computing the mean velocity and flow depth of the current. The boundary condition imposed at the front leads to technical mathematical difficulties. Indeed, unlike in the Boussinesq case, no regular solution to the shallow-water equations satisfies the downstream condition, but when the flow is supercritical at the channel inlet, it is possible to construct a piecewise solution by patching a regular solution to an exceptional solution, which represents the head behavior. To better understand this result and make sure that the result is physically relevant, we consider the Navier-Stokes equations within the high-Reynolds-number limit. Approximate similarity solutions can be worked out, which support our earlier analysis on the shallow-water equations. While the flow body is self-similar and weakly rotational, the head is not self-similar, but tends toward a self-similarity shape at long times. It is characterized by a strong vorticity, a straight free surface, and a nonuniform velocity profile, which becomes flatter and flatter with time.
\end{abstract}

(C) 2006 Elsevier B.V. All rights reserved.

Keywords: Shallow-flow equations; Non-Boussinesq current; Front; Gravity current; Similarity solutions; Euler equations

\section{Introduction}

Lubrication theory has been extensively used to model industrial and natural flows such as coating films [49,64], water waves [60], atmospheric flows [59], snow avalanches, and debris flows $[3,37]$. Within this theory, the equations of motion take the form of a set of hyperbolic partial differential equations, which govern the spatial and temporal variations in the flow depth and mean velocities. These governing equations are usually derived by taking the flow depth average of the local mass and momentum balance equations and by assuming that the streamwise length scale outweighs the vertical scale. Typical examples of governing equations derived within this framework include the shallow-water equations [56], the

\footnotetext{
* Corresponding author. Tel.: +41 21 6933287; fax: +41 2169336767.

E-mail address: christophe.ancey@epfl.ch (C. Ancey).
}

nonlinear diffusion equation for creeping Newtonian and non-Newtonian flows $[5,34,41,48,50]$, and the Savage-Hutter equations for fast-flowing granular materials [57].

A particularity of many free-surface flows is that they have a front, where one would ideally like to impose a vanishing flow depth. Fig. 1(a) shows a typical example of a gravity current with a front. The existence of a front implies a number of difficulties from the physical and mathematical points of view, which have led to several paradoxes. For instance, for lubrication films, taking into account surface tension in the governing equations makes the stress distribution singular at the front point; furthermore, the no-slip condition imposed along solid boundaries conflicts with the need for the fluid edge to advance. To avoid these issues, a more refined description of the physical processes involved in the contact-line problem may be needed, but has the disadvantage of significantly increasing the level of complexity. Instead, computational tricks are often preferred. A classic method is to assume that the plane along 

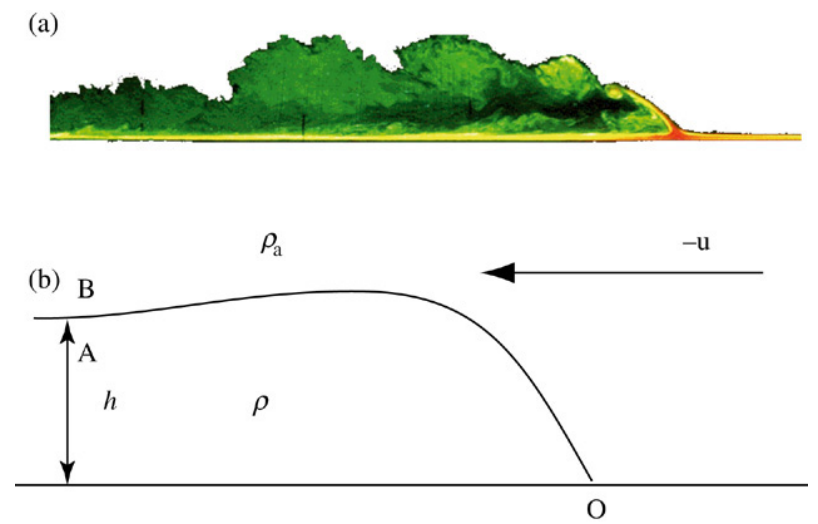

Fig. 1. (a) Front of a gravity current produced by unleashing a brine solution in a water tank. (b) Idealization of a gravity current. Point A lies within the current of heavy fluid, while point B is located along the streamline formed by the interface between the current and the surrounding fluid, in the close neighborhood of A.

which the fluid spreads out is pre-wetted by a thin layer of fluid so that the flow depth drops not to zero at the front, but to a small value $\varepsilon$. Yet even with this change in the boundary condition at the front, there are severe technical mathematical difficulties that arise when $\varepsilon \rightarrow 0$ and make the solution nonphysical [61].

Another example is provided by gravity currents such as the one shown in Fig. 1(a). A gravity current is produced by releasing fluid within an ambient fluid of lower density $\rho_{a}$. At sufficiently high Reynolds numbers, the viscous dissipation is negligible and the current dynamics is controlled by the balance between buoyancy and inertia. For this case, van Kármán [62] and Benjamin [7] showed that a steady current of uniform flow depth $h$ is preceded by a leading edge, the velocity of which is constant and given by

$\frac{u}{\sqrt{g^{\prime} h}}=F r$

where $F r$ is the Froude number, $g^{\prime}=\Delta \rho / \rho_{a}$ is the reduced gravity, and $\Delta \rho$ is the density contrast between the current and the ambient fluid. This condition was derived by van Kármán [62] by considering an inviscid gravity current moving at constant velocity in a stagnant, infinitely deep medium of density $\rho_{a}$ [see Fig. 1(b)]: in a coordinate system moving at the same velocity as the head, the surrounding fluid arrives from the right at velocity $-u$. Van Kármán applied the Bernoulli equation to a streamline $\mathrm{OB}$ and an equilibrium condition at point A. Note that the streamline and the interface coincide in a steady state. Both conditions can also be applied to the stagnation point $\mathrm{O}$, where the velocity vanishes. We refer to $P_{\mathrm{A}}, P_{\mathrm{B}}$, and $P_{\mathrm{O}}$ as the dynamic pressures at point $\mathrm{A}, \mathrm{B}$, and $\mathrm{O}$, respectively. Van Kármán obtained the relations $P_{\mathrm{B}}+\rho_{a} u^{2} / 2+$ $\rho_{a} g h=P_{\mathrm{O}}$ and $P_{\mathrm{A}}+\rho g h=P_{\mathrm{O}}$. Then, using the pressure equilibrium condition at the interface $P_{\mathrm{A}}=P_{\mathrm{B}}$, he derived Eq. (1). His derivation was not without criticism [e.g., see [39] for an overview], but the result was widely recognized as valid.
Since the front velocity can also be defined as the time derivative of the front position $u=\dot{x}_{f}$, Eq. (1) is equivalent to imposing a finite flow depth at the front, which adjusts to the front velocity

$h=\frac{\dot{x}_{f}^{2}}{g^{\prime} F r^{2}}$.

This result, primarily derived for steady uniform currents, was then applied to all flow conditions by considering that Eq. (1) provides the boundary condition at the front. There are still avid debates on the relevance and physical meaning of a Benjamin-like boundary condition (1), especially when it is applied to time-dependent flows $[39,44]$. Eq. (1) means that to a large extent, the current dynamics is controlled by the leading edge whatever the flow regime $[33,35]$. With a Benjamin-like relation as the downstream boundary condition, the shallowwater equations exhibit a wide class of similarity solutions $[21,25,32,35,53]$. There are, however, very few experimental investigations that quantitatively checked the reliability of these solutions $[44,45]$. A major source of difficulties stems from the complexity of the flow in real gravity currents at high Reynolds numbers. As shown in Fig. 1 in the case of a laboratory experiment, the flow depth profile is irregular and unsteady because of the intense mixing, density stratification, and free-surface instabilities occurring within the leading edge. By systematically comparing the theoretical and experimental flow depth profiles, Marino et al. [44] concluded that for a gravity current in a similarity phase, the Froude number in Eq. (1) is not constant, but varies with the head Reynolds number.

The objective of this paper is to examine the existence and features of similarity solutions for gravity currents, which are in the high-Reynolds-number limit and characterized by large density differences with the ambient fluid. This flow regime is often referred to as the non-Boussinesq regime and shallowwater equations can be used to describe it. The previous arguments supporting the idea of a finite flow depth at the front no longer hold for a non-Boussinesq regime since the resisting effect of the ambient fluid becomes negligible. A direct consequence is that the downstream boundary condition is now given by

$h=0$.

A number of natural and non-natural flows such as flash floods induced by a dam break [14,28,52], snow avalanches [2], and pyroclastic clouds [17] provide typical examples. In the laboratory, non-Boussinesq currents are usually generated by suddenly releasing a homogeneous fluid of low viscosity or a suspension of particles in the air. Experiments of this kind remain difficult to carry out and require very long flumes. Surprisingly, numerical solutions are also extremely difficult to compute because of the difficulties induced by the free surface and front (e.g., front tracking, instabilities, contact line, mass conversation with some numerical techniques) $[16,18]$. In this context, seeking analytical solutions is of paramount importance both for gaining insight into the flow dynamics 
and for testing numerical models. Various solutions have been worked out using different techniques: the method of characteristics $[14,52,66]$, the hodograph transformation [13, 20], and self-similar solutions [25,26]. Similarity solutions are here of particular relevance in the present context since they embody the inertia-buoyancy balance, which is anticipated to be the driving mechanism in the flow dynamics when the influence of the initial conditions becomes negligibly small. Earlier investigations concentrated on the similarity solutions for the Boussinesq regime. Gratton and Vigo [25] stated that the non-Boussinesq boundary condition (3) is retrieved by making the Froude number $F r$ tend to infinity in Eq. (2). In a recent paper [4], however, we have shown that this asymptotic limit poses severe mathematical difficulties since the only regular self-similar solution to the shallow-water equation is the trivial solution $h=0$ for the entire domain of flow. An exceptional solution may, however, be worked out on some occasions.

In this paper, we further examine this issue by systematically seeking similarity solutions to the shallow-water equations. After briefly recalling the physical setting and related equations of motion in Section 2 and the boundary value problem for Boussinesq and non-Boussinesq currents in Section 3, we address the specific problem of the existence of similarity solutions in Section 4. In these sections, emphasis is given to the understanding of the solution behavior; the mathematical details are thus deferred to appendices. We shall see that similarity solutions exist solely when the current is abundantly supplied in fluid by a source and the fluid flows sufficiently fast for the flow body to be in a supercritical regime (i.e., Froude number in excess of unity). These existence conditions contrast substantially with those obtained by Gratton and Vigo [25] for the Boussinesq regime. In order to elucidate this point, we will refine our analysis by working out similarity solutions to the Navier-Stokes equations in the high-Reynolds-number limit. Because of the curvature of the free surface, we shall consider the body and the head separately. In Section 5, we shall see that for the body of supercritical flows, similarity solutions can be constructed, which are in fairly good agreement with those obtained for the shallow-water equations. Unfortunately, these similarity solutions are not valid within the leading edge and this region is strongly resistant to similarity analysis. Approximate analytical methods will be used in Section 6 to derive the salient features. By expanding the velocity into a time series, we will notably show that at long times, the leading edge is characterized by a straight free surface and a significant source of vorticity.

\section{Physical setting and governing equations}

\subsection{Flow geometry}

Let us consider a shallow layer of fluid flowing over a rigid horizontal impermeable plane. The fluid is incompressible and homogeneous (i.e., no density stratification); its density is denoted by $\rho$. Its dynamic viscosity is denoted by $\mu$. A twodimensional flow regime is assumed, namely any cross-stream variation is neglected. The depth of the layer is given by $h(x, t)$ (see Fig. 2). The flow is generated by a source of fluid: at $t=0$, the sluice gate at the inlet is raised with a given aperture rate $h_{0}(t)$, specified below. Ahead of the front, there is a dry bed. The surrounding fluid (assumed to be air) is taken to be inviscid and of low density, and therefore dynamically passive. Surface tension is neglected.

\subsection{Flow depth averaged equations}

The local governing equations are given by the shallowwater equations, the dimensionless form of which is $[60,66]$

$$
\begin{aligned}
& \frac{\partial h}{\partial t}+\frac{\partial h \bar{u}}{\partial x}=0, \\
& \frac{\partial h \bar{u}}{\partial t}+\frac{\partial h \overline{u^{2}}}{\partial x}+h \frac{\partial h}{\partial x}=0,
\end{aligned}
$$

where the bar refers to flow depth averaged values: $\bar{u}(x, t)=$ $h^{-1} \int_{0}^{h} u(x, y) \mathrm{d} y$, where $u(x, y, t)$ denotes the horizontal velocity field. The dimensionless velocity, flow depth, distance, and time were defined as $\bar{u}=\hat{u} / U_{*}, h=\hat{h} / H_{*}, x=$ $\hat{x} / L_{*}$, and $t=\hat{t} U_{*} / L_{*}$, respectively. In these equations, the hat refers to dimensional variables, whereas the star refers to typical scales: $H_{*}$ and $L_{*}$ are the vertical and horizontal length scales; $U_{*}=\sqrt{g H_{*}}$ is the velocity scale. By introducing the Boussinesq coefficient $\gamma$, we can relate the mean square velocity to the square of the mean velocity: $\overline{u^{2}}=\gamma \bar{u}^{2}$. The Boussinesq coefficient reflects the shear in the vertical profile of the horizontal fluid velocity. When $\gamma=1$, there is no shear in the vertical profile of the streamwise velocity, whereas $\gamma>1$ means that there is shear. Assuming that $\gamma$ is a known free parameter, we obtain a closed set of equations for $h$ and $\bar{u}$ :

$$
\begin{aligned}
& \frac{\partial h}{\partial t}+\frac{\partial h \bar{u}}{\partial x}=0, \\
& \frac{\partial \bar{u}}{\partial t}+(2 \gamma-1) \bar{u} \frac{\partial \bar{u}}{\partial x}+\bar{u}^{2} \frac{\partial \gamma}{\partial x}=-\frac{\partial h}{\partial x}\left(1+\frac{\bar{u}^{2}}{h}(\gamma-1)\right) .
\end{aligned}
$$

When $\gamma$ is set equal to unity in the momentum balance equation (5), we retrieve the usual form of the shallow-water equations [60]. When $\gamma$ is constant and in excess of unity, the convective acceleration term is weighted by the shape factor $2 \gamma-1$, while a Chézy-like term affects the pressure gradient.

\subsection{Boundary conditions}

At the source $x=0$, the boundary conditions are given by a flux condition in the form

$\bar{u} h=n A t^{n-1}$,

with $n$ a prescribed coefficient and $A$ a constant, and a condition on the sluice-gate aperture

$h=h_{0}(t)=\beta t^{m}$,

where $m=\frac{2}{3}(n-1)$ and $\beta$ is another constant. According to Gratton and Vigo [25], $n$ must lie within the range $0 \leq n \leq 4$; 


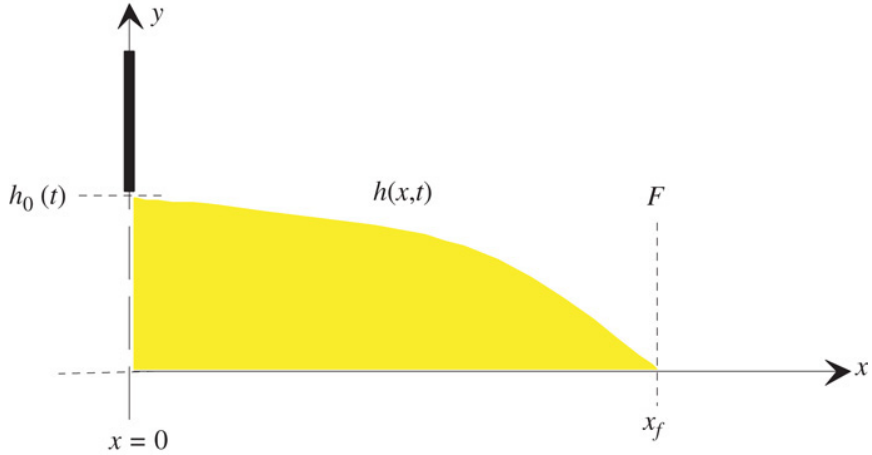

Fig. 2. The configuration of the flow.

this condition on $n$ is needed for the shallowness assumption to be consistent. Note that the boundary condition (6) is equivalent to imposing the volume growth rate at the source: $\mathcal{V}=\int_{0}^{x_{f}} h(x, t) \mathrm{d} x=A t^{n}$, and a constant Froude number

$F r_{0}=\frac{\bar{u}}{\sqrt{h}}=\alpha$,

where $\alpha$ is a constant satisfying $\alpha \beta^{3 / 2}=n A$.

The other boundary conditions are prescribed at the front. For a gravity current in a non-Boussinesq regime, the front position $x_{f}$ is the point where the flow depth drops to zero:

$h_{f}(t)=h\left(x_{f}, t\right)=0$.

Moreover, the front velocity is

$\bar{u}\left(x_{f}\right)=\dot{x}_{f}$,

where the dot denotes the time derivative.

\subsection{Jump conditions}

The solutions to the system (4) and (5) may admit discontinuities (called shock or hydraulic jumps in the hydraulic literature). The flows either side of a discontinuity are connected by jump conditions, which express conservation of mass and momentum across the moving discontinuity. Denoting the shock speed by $s$, we can write these jump conditions associated with the conservative form of Eqs. (4) and (5) as follows [66]:

$\llbracket h \bar{u} \rrbracket=s \llbracket h \rrbracket$,

$\llbracket \gamma h \bar{u}^{2}+h^{2} / 2 \rrbracket=s \llbracket h \bar{u} \rrbracket$,

where the $\llbracket \cdot \rrbracket$ denotes the difference upstream and downstream of the shock.

\section{Similarity solutions for gravity currents}

\subsection{Similarity variables and functions}

To gain insight into the behavior of solutions to the shallowwater equations, a classic approach is to seek similarity solutions. Ritter [52] worked out a similarity solution for the so-called dam-break problem, where an infinite volume of fluid is suddenly released on a smooth horizontal plane. Grundy and Rottman [27] showed that the shallow-water equations admit stable similarity solutions and used the phase-plane formalism to construct solutions that are more general than Ritter's solution. Gratton and Vigo [25] elaborated on this method to take shock occurrence and upstream boundary conditions into account. Following these authors, we impose

$\bar{u}=\delta \xi t^{\delta-1} V(\xi)$,

$h=\delta^{2} \xi^{2} t^{2(\delta-1)} Z(\xi)$,

with $\xi=x / t^{\delta}$ the similarity variable. The rationale for these expressions is outlined in Appendix A. The boundary condition at the source (6) implies that the parameter $\delta$ is related to the volume-growth-rate exponent $n$

$\delta=\frac{2+n}{3}$.

Note that for $\delta>1$, the front $\left(\xi_{f}=\xi_{f} t^{\delta}\right)$ accelerates, whereas it decelerates for $\delta<1$. The particular case $\delta=1$ corresponds to a constant inflow and the corresponding solution is usually referred to as the dam-break solution since it coincides with the solution derived by Ritter [52].

We substitute the forms (13) and (14) into the governing equations (4) and (5) to end up with a single first-order ordinary differential equation

$\frac{\mathrm{d} Z}{\mathrm{~d} V}=\frac{F(V, Z)}{G(V, Z)}$

where $F(V, Z)=-Z(2 Z \delta+V(-2 V \delta \gamma+4 \gamma+3 \delta-3)-2)$ and $G(V, Z)=Z(2-(V+2) \delta)+V(V(2 \gamma+((V-4) \gamma+$ $3) \delta-3)+1)$. The full derivation of this equation is recalled in Appendix A.

\subsection{Boussinesq regimes}

The early papers were devoted to planar inviscid Boussinesq gravity currents, i.e., currents for which the Reynolds number is sufficiently high for the viscous dissipation to be negligible, while the resistance action of the ambient fluid implies a Benjamin-like condition

$Z=Z_{f} \quad$ and $\quad V=1$,

at the front $\left(\xi=\xi_{f}\right)$ [25-27]. These conditions have been derived from Eqs. (9) and (10), the only difference being that now at the front, the flow depth is finite $\left(Z=Z_{f}>0\right)$ and the corresponding Froude number $F r_{f}=V / \sqrt{Z}=1 / \sqrt{Z_{f}}$ remains constant.

In this case, Gratton and Vigo [25] demonstrated the existence of similarity solutions for a wide range of $Z_{f}$. For Boussinesq gravity currents, the main issue lies in the proper determination of the front depth [44]. The similarity solution may admit discontinuities because near the source, the flow can be either supercritical $\left(F r_{0}>1\right)$ or subcritical $\left(F r_{0}<1\right)$, while close to the front, the flow is subcritical or transcritical. 


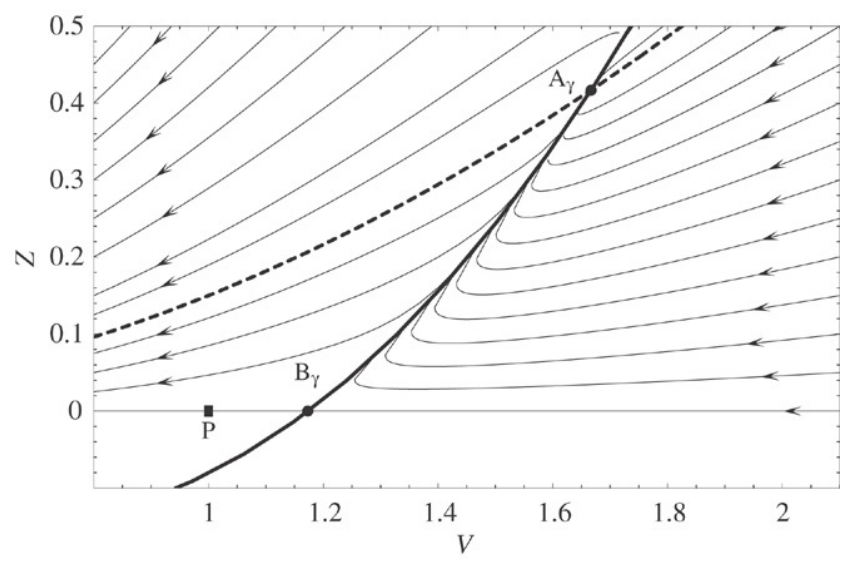

Fig. 3. Integral paths (solid lines) in the close neighborhood of the front point $P$. The thick solid line represents the separatrix $S_{\mathrm{AB}}$ of equation $Z=5 / 12+8(V-$ $5 / 3) / 7+843(V-5 / 3)^{2} / 1372+6966(V-5 / 3)^{3} / 420175+O\left(V^{3}\right)$. The thick dashed line represent the special solution $Z=(9-8 \gamma) V^{2} / 4$. Computations made for $n=5 / 2$ and $\gamma=1.05$.

\subsection{Non-Boussinesq regimes}

The case of non-Boussinesq gravity currents has received far less attention. When a current is in a non-Boussinesq regime and at high Reynolds numbers, the density mismatch between the current and the ambient fluid is so weak that the resisting effect of the ambient fluid becomes negligible and one expects the Benjamin-like condition (16) at the front to no longer hold and to require replacing with

$Z=0 \quad$ and $\quad V=1$,

which is the equivalent of Eqs. (9) and (10) in terms of similarity variables. In their analysis, Gratton and Vigo [25] stated that the non-Boussinesq boundary condition (i.e., vanishing flow depth at the front) can be retrieved from the Boussinesq case by making the Froude number at the front tend to infinity. However, from a purely mathematical point of view, the boundary value problem remains ill-posed since the only regular similarity solution that vanishes at the front is the trivial solution (i.e., flow depth zero everywhere).

To understand the situation, let us take a closer look at Fig. 3, which shows a few integral paths in the phase plane $V-Z$ in the vicinity of the front. On this figure, point $\mathrm{P}(V=1, Z=$ 0 ) represents the front. The integral paths were obtained by numerically integrating Eq. (15) for various initial conditions. They are represented by thin solid lines oriented with arrows showing the direction of increasing $\xi$. No curve passes through point $\mathrm{P}$, except for the trivial solution $Z=0$. Also on this figure, we reported two thick (solid and dashed) lines, which represent special solutions. These curves intersect at point $\mathrm{A}_{\gamma}$, which is a singular point (node); we will return to these special curves later on.

More recently, Ruo and Chen [54] suggested that the boundary condition at the front for non-Boussinesq currents is a part of the problem to be solved, but they were able to derive this condition solely for constant-inflow currents. Montgomery and Moodie [47] modified the governing equations to transform them into an initial value problem, but they did not provide similarity solutions.

\section{Existence of similarity solutions for non-Boussinesq currents}

We want to determine the solution to Eq. (15) for nonBoussinesq currents, i.e., we consider that the boundary conditions at the front are given by Eq. (17). The boundary conditions at the source are given by Eq. (9) for the physical variables. Translated to a form in terms of similarity variables, these conditions imply that at the source, we have

$Z \propto \frac{\beta}{\delta^{2} \xi^{2}} \quad$ and $\quad V \propto \frac{\alpha \sqrt{\beta}}{\delta \xi} \quad$ when $\xi \rightarrow 0$.

These relations mean that in the phase plane, the source point $\mathrm{S}$ lies at infinity on a parabola of equation $Z=(V / \alpha)^{2}$, while the front point $\mathrm{P}$ is a fixed point of coordinates $(1,0)$. Solving Eq. (15) with these boundary conditions boils down to finding an integral path or a piecewise integral path (i.e., made up of pieces borrowed from different paths), which links point $S$ to point $\mathrm{P}$.

Here, we explain how to construct similarity solutions by exploiting the topological features of the integral curves close to the front point.

\subsection{Topological features of the phase plane}

Here we wish to plot and describe the integral paths, which represent the solutions to Eq. (15) in the first quadrant $(V \geq 0$, $Z \geq 0$ ). The topological features of these curves are dictated by three elements: (i) the position and shape of the critical curves $F=0$ and $G=0$, (ii) the position and nature of the singular points, and (iii) the position of the separatrices and exceptional solutions emanating from the singular points. We will review these different features to better understand the behavior of the solution curves in the close neighborhood of the front.

The equation $F(V, Z)=0$ gives rise to two curves: the $V$-axis $(Z=0)$ and a parabola called $C_{F}$ of equation

$Z=\frac{2 \gamma \delta V^{2}+(-4 \gamma-3 \delta+3) V+2}{2 \delta}$,

which is called the nullcline. When an integral path crosses $C_{F}$, its tangent at the point of intersection is horizontal. Similarly, the equation $G(V, Z)=0$ provides a rational function of $V$

$Z=\frac{V\left(\gamma \delta V^{2}+(-4 \delta \gamma+2 \gamma+3 \delta-3) V+1\right)}{(V+2) \delta-2}$,

which defines the curve called $C_{G}$.

When an integral path crosses $C_{G}$, its tangent at the point of intersection is vertical. Fig. 4 reports the curves $F=0$ and $G=$ 0 for the particular case $\delta=5 / 2$ and $\gamma=1.05$. We have also reported a third critical curve $C_{I}$, which is the locus of points at which $V^{\prime}(\xi)$ and $Z^{\prime}(\xi)$ diverge; for simplicity, since this curve is not indispensable to understanding the fundamental features of the phase plane, we defer its analysis to Appendix B. Still in Fig. 4, we have drawn two integral paths to show their 


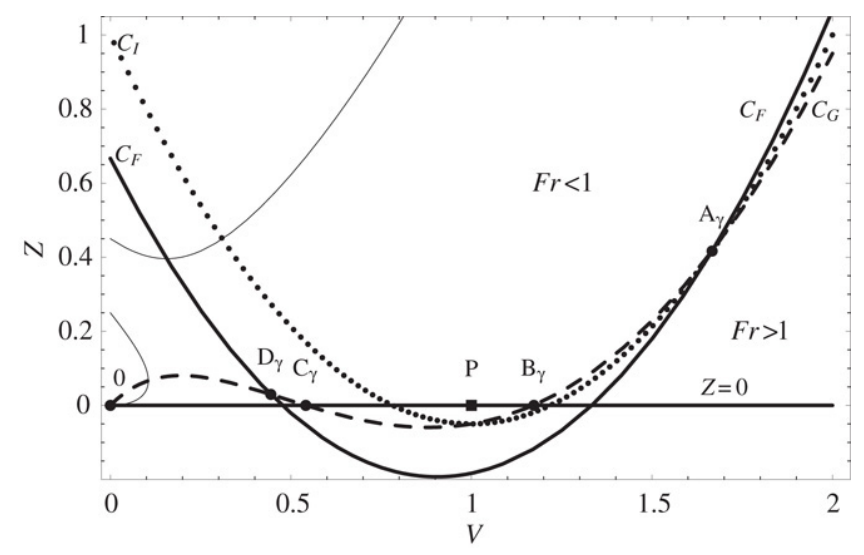

Fig. 4. Position of the critical curves $C_{I}$ (dotted line), $C_{G}$ (dashed line), and $C_{F}$ (bold line). Point $\mathrm{P}$ (front) is represented by a black rectangle. The singular points $\mathrm{A}_{\gamma}, \mathrm{B}_{\gamma}, \mathrm{C}_{\gamma}, \mathrm{D}_{\gamma}$, and $\mathrm{O}$ are also represented (labeled dots). The two thin solid lines represent integral paths. Computations are made for $n=5 / 2$ and $\gamma=1.05$.

behavior when they cross the critical curves. Note that for $V>1$, the critical curves $C_{F}$ and $C_{G}$ are relatively close, which implies that in this region of the phase plane, the integral paths experience significant changes in their shape.

In the phase plane, the singular points are the points at which $G$ and $F$ vanish simultaneously [6]. The equation $F=G=0$ yields five finite singular points referred to as $\mathrm{O}$ (origin point), $\mathrm{A}_{\gamma}, \mathrm{B}_{\gamma}, \mathrm{C}_{\gamma}$, and $\mathrm{D}_{\gamma}$. Appendix $\mathrm{B}$ recalls the fundamental properties of singular points. Fig. 5 reports the five singular points for the particular cases $\delta=3 / 2$ (accelerating front, $n=5 / 2$ ) and $\delta=5 / 6$ (decelerating front, $n=1 / 2$ ). Table 1 summarizes their properties. These properties change significantly with $\delta$. On the whole, we have to separately consider the cases $\delta>1$ (accelerating front), $\delta<1$ (decelerating front), and $\delta=1$ (front at constant velocity).

With each singular point, we can associate two specific curves called separatrices, which can be interpreted as special or exceptional solutions to Eq. (15) and local symmetry axes in the phase plane. Given the density of singular points in the lower left corner of the first quadrant, the singular points can share the same separatrices and this can be used as a convenient way to label these curves. For instance, separatrix $S_{\mathrm{AB}}$ is the curve that relates the singular points $\mathrm{A}_{\gamma}$ and $\mathrm{B}_{\gamma}$. In Fig. 5, the separatrices are plotted as dashed curves. For $\delta>1$, separatrix $S_{\mathrm{AB}}$ is the asymptotic curve toward which all the curves passing through the node $\mathrm{A}_{\gamma}$ tend when approaching this point; the sole exception is the separatrix $S_{\mathrm{AD}}$ (see Appendix B). Separatrix $S_{\mathrm{AB}}$ is also the only nontrivial integral path passing through the saddle point $\mathrm{B}_{\gamma}$ [see Fig. 5(a)]. For $\delta<1$, the roles of $\mathrm{A}_{\gamma}$ and $\mathrm{B}_{\gamma}$ are interchanged: $S_{\mathrm{AB}}$ is the asymptotic curve for the node $\mathrm{B}_{\gamma}$, while it is an exceptional solution for the saddle point $\mathrm{A}_{\gamma}$ [see Fig. 5(b)]. A similar interpretation can be made for separatrices $S_{\mathrm{BC}}$ and $S_{\mathrm{CD}}$ and thus is not repeated here.

Once the critical curves, the singular points, and the separatrices are established, we can outline the behavior of solutions within the tip region. Numerically integrating Eq. (15) with various boundary conditions makes it possible to plot a few integral paths, which illustrate the rich behavior of solutions in
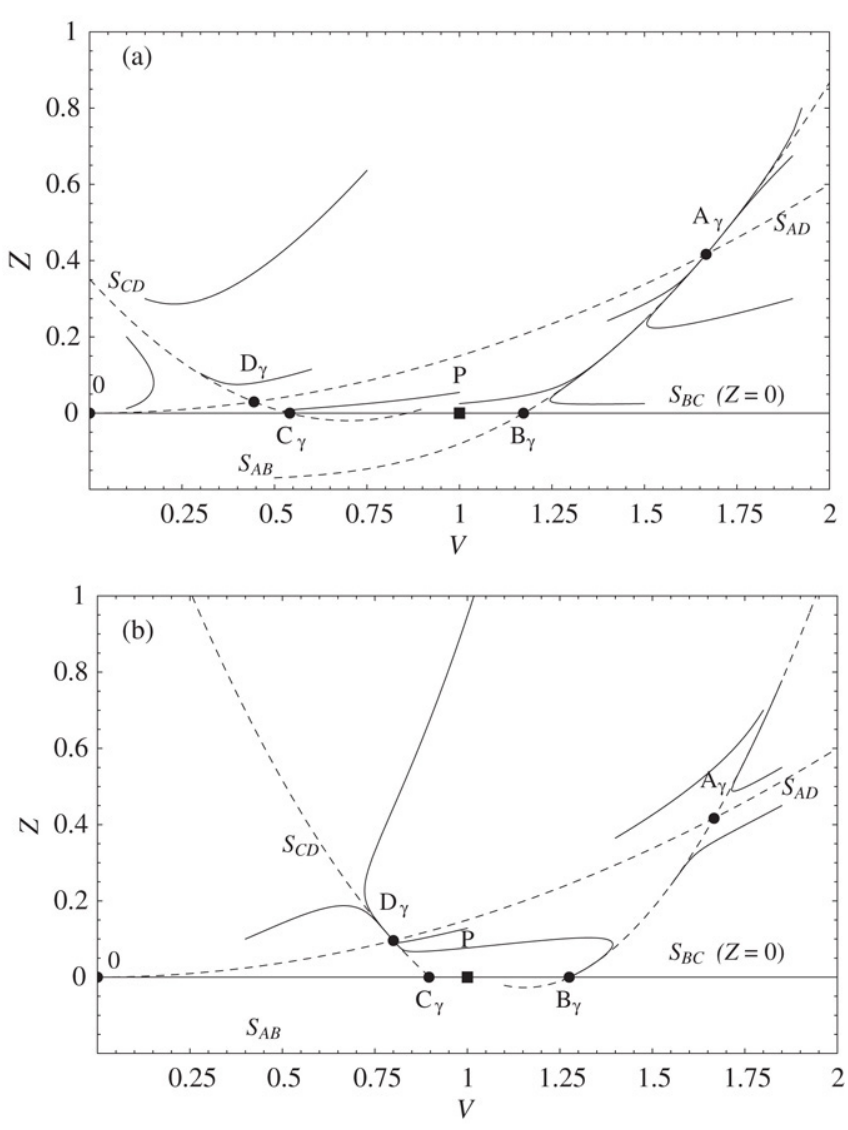

Fig. 5. Positions of the singular points $\mathrm{A}_{\gamma}, \mathrm{B}_{\gamma}, \mathrm{C}_{\gamma}, \mathrm{D}_{\gamma}$, and $\mathrm{O}$ (block dots) and their separatrices (dashed lines). Thin solid lines represent integral paths. The singular points are presented by labeled dots. Point $\mathrm{P}$ (front) is represented by a black rectangle. Computations made for $\gamma=1.05$ with (a) $\delta=3 / 2$ and (b) $\delta=5 / 6$.

the immediate neighborhood of the front. Fig. 6 shows different phase portraits corresponding to an accelerating front $(\delta=3 / 2$ or $n=5 / 2)$, a front at constant velocity $(\delta=1$ or $n=1)$, and a decelerating front $(\delta=5 / 6$ or $n=1 / 2)$ for the particular case $\gamma=1.05$. For completeness, all the special curves, which have been discussed earlier (i.e., critical curves and separatrices) have also been plotted in the phase planes. The same exercise is repeated in Fig. 7 when the Boussinesq coefficient drops to unity. On the whole, there are small differences. The most noticeable feature is the front point $\mathrm{P}$ becoming singular when $\gamma=1$ : it coincides with either $\mathrm{B}_{\gamma}$ or $\mathrm{C}_{\gamma}$.

\subsection{Construction of physically admissible solutions for non- Boussinesq regimes}

When $\gamma>1$, i.e., when there is shear in the vertical direction of the horizontal velocity field, point $\mathrm{P}$ is a regular point. Apart from the trivial solution $Z=0$, no regular solution or exceptional solution passes through it. As seen in Fig. 6 for various $\delta$ values, a bundle of paths approach point $\mathrm{P}$, but never reach it. We conclude that there is no continuous similarity solution to Eq. (15) when $\gamma>1$.

When $\gamma=1$, i.e., the velocity profile in the vertical is uniform, point $\mathrm{P}$ is singular and coalesces with point $\mathrm{B}_{1}$ for 
Table 1

Positions and properties of the finite singular points of Eq. (15) in the first quadrant of the phase plane

\begin{tabular}{|c|c|c|c|}
\hline Singular points & Coordinates & Case & Type \\
\hline $\mathrm{O}$ & $(0,0)$ & & Node \\
\hline $\mathrm{A}_{\gamma}$ & $\left(\frac{2}{4 \gamma-3}, \frac{9-8 \gamma}{(4 \gamma-3)^{2}}\right)$ & $\begin{array}{l}0<\delta \leq(4 \gamma-3) / 3 \\
(4 \gamma-3) / 3<\delta<1 \\
1 \leq \delta\end{array}$ & $\begin{array}{l}\text { Node } \\
\text { Saddle } \\
\text { Node }\end{array}$ \\
\hline $\mathrm{B}_{\gamma}$ & $\left(\frac{K+\sqrt{K^{2}-4 \gamma \delta}+3}{2 \gamma \delta}, 0\right)$ & $\begin{array}{l}0<\delta \leq 1 \\
\delta>1\end{array}$ & $\begin{array}{l}\text { Node } \\
\text { Saddle }\end{array}$ \\
\hline $\mathrm{C}_{\gamma}$ & $\left(\frac{K-\sqrt{K^{2}-4 \gamma \delta+3}}{2 \gamma \delta}, 0\right)$ & $\begin{array}{l}0<\delta<1 \\
\delta \geq 1\end{array}$ & $\begin{array}{l}\text { Saddle } \\
\text { Node }\end{array}$ \\
\hline $\mathrm{D}_{\gamma}$ & $\left(\frac{2}{3 \delta}, \frac{9-8 \gamma}{9 \delta^{2}}\right)$ & $\begin{array}{l}0<\delta<(4 \gamma-3) / 3 \\
(4 \gamma-3) / 3 \leq \delta \leq 1 \\
\delta>1\end{array}$ & $\begin{array}{l}\text { Saddle } \\
\text { Node } \\
\text { Saddle }\end{array}$ \\
\hline
\end{tabular}

We introduced $K=4 \delta \gamma-2 \gamma+3 \delta-3$.

$\delta>1$ or $\mathrm{C}_{1}$ for $\delta<1$ (see Fig. 7); for $\delta=1$, the three points $\mathrm{P}, \mathrm{B}_{1}$, and $\mathrm{C}_{1}$ coincide. For both $\delta>1$ and $\delta<1$, point $\mathrm{P}$ is a saddle and apart from the trivial solution $Z=0$, the only integral path arriving at $\mathrm{P}$ is the separatrix $S_{\mathrm{AB}}$ for $\delta>1$ [see Fig. 7(a)] or $S_{\mathrm{CD}}$ for $\delta<1$ [see Fig. 7(c)]. This means that for $\gamma=1$, it is possible to find a solution to Eq. (15) and this solution is necessarily an exceptional solution, which cannot be obtained by direct numerical integration techniques (see Appendix B).

For the separatrices to be full solutions to our boundary value problem, they must also satisfy the boundary conditions at the source (18). Dominant-balance analysis shows that when $Z$ and $V$ tend together toward infinity, the regular integral paths behave as $Z \propto V^{2}$ and so the exceptional solution $S_{\mathrm{AB}}$ does too. This means that it is possible to find an $\alpha$ value such that the curve $S_{\mathrm{AB}}$ asymptotically patches onto a parabola of equation $Z=(V / \alpha)^{2}$, which shows that it satisfies the boundary conditions (18). In contrast, when $Z$ tends toward infinity while $V$ comes close to zero, the integral paths behave as $Z \propto V^{-1}$, which implies that the separatrix $S_{\mathrm{CD}}$ cannot satisfy the boundary conditions (18).

Using the properties of the phase plane, it is possible to construct piecewise solutions. Indeed, whenever an integral path reaches a node, we can take another integral path, which means that every piece of integral path between nodes is a potential local solution to our boundary value problem. To illustrate this technique, let us start with the Ritterlike solutions, which can be derived fully analytically (see Appendix D).

The Ritter-like solutions are the solutions pertaining to $\delta=n=1$ (constant-velocity front). As shown in Fig. 8, a remarkable trait of the phase portrait is that the three critical curves $C_{F}, C_{G}$, and $C_{I}$, together with the two separatrices $S_{\mathrm{AB}}$ and $S_{\mathrm{CD}}$, coincide and form a parabola, the vertex of which lies at the front point P. This means that any piece of this curve may be a part of the solution sought since it is the only nontrivial solution passing through P. Another noticeable
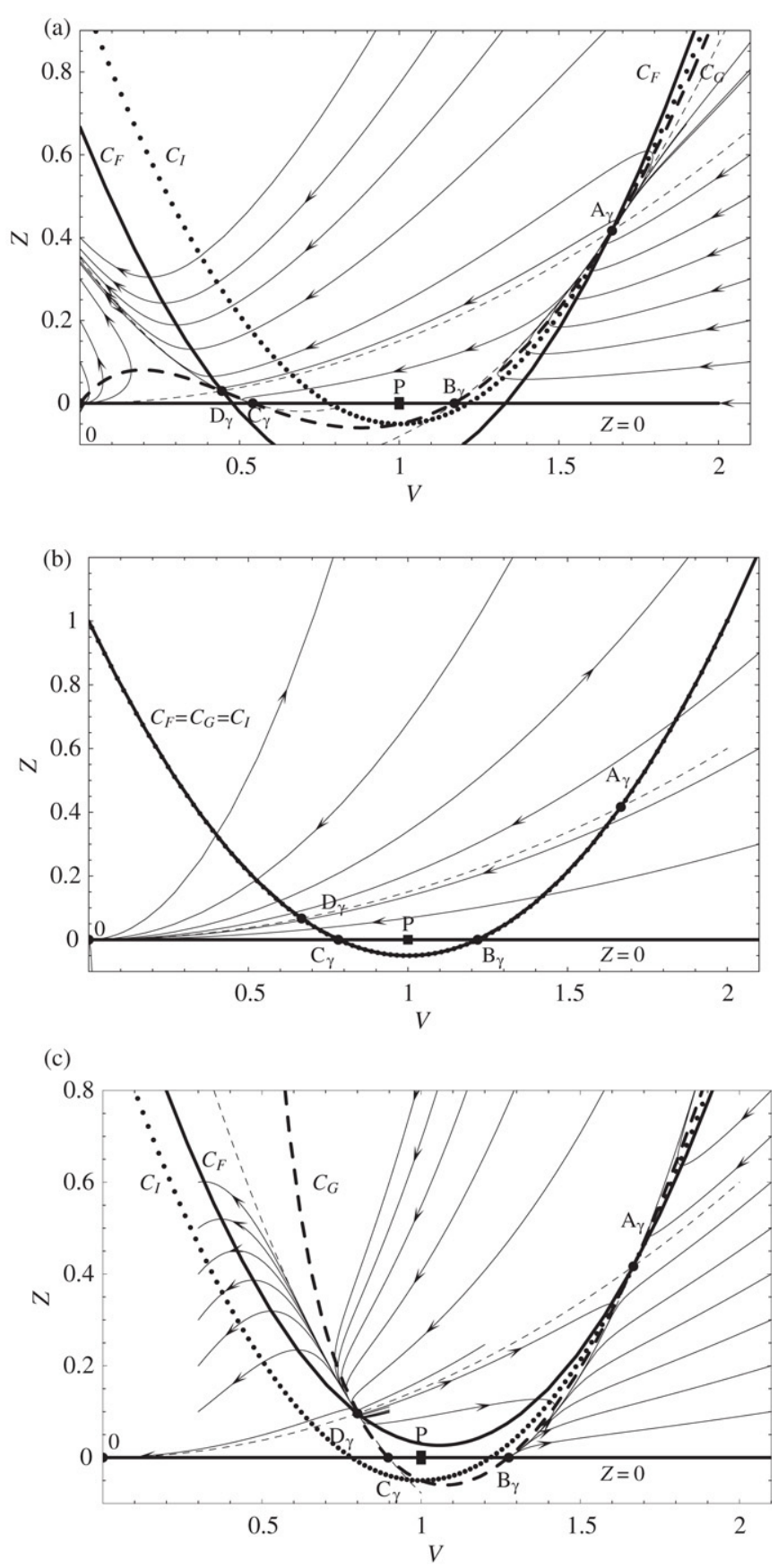

Fig. 6. Phase portraits for $\gamma=1.05$, (a) $\delta=3 / 2(n=5 / 2)$, (b) $\delta=1(n=1)$, and (c) $\delta=5 / 6(n=1 / 2)$. For each figure, we took $\gamma=1.05$. The solid line $C_{F}$ (bold line) represents the locus of points where $F=0$, while the curve $C_{G}$ (dashed line) is given by the equation $G(V, Z)=0$. The critical curve $C_{I}$ (dotted line) is also plotted. The thin lines represent the integral paths, while the thin dashed lines represent the separatrix of the singular points.

feature is the singular points $B_{1}$ and $C_{1}$ coinciding with $P$. We have to distinguish flows with supercritical and subcritical flow conditions at the source. In the former case $(\alpha>1)$, the solution curve is made up of two pieces in the phase plane. Indeed, any integral path emanating from the source point $S$ goes toward the origin point $\mathrm{O}$ without passing through $\mathrm{P}$, but it intersects the parabola $C_{I}$ at point E (see Fig. 8). The path $\mathrm{E} \rightarrow \mathrm{P}$ on $C_{I}$ is thus the second piece of the solution. See 

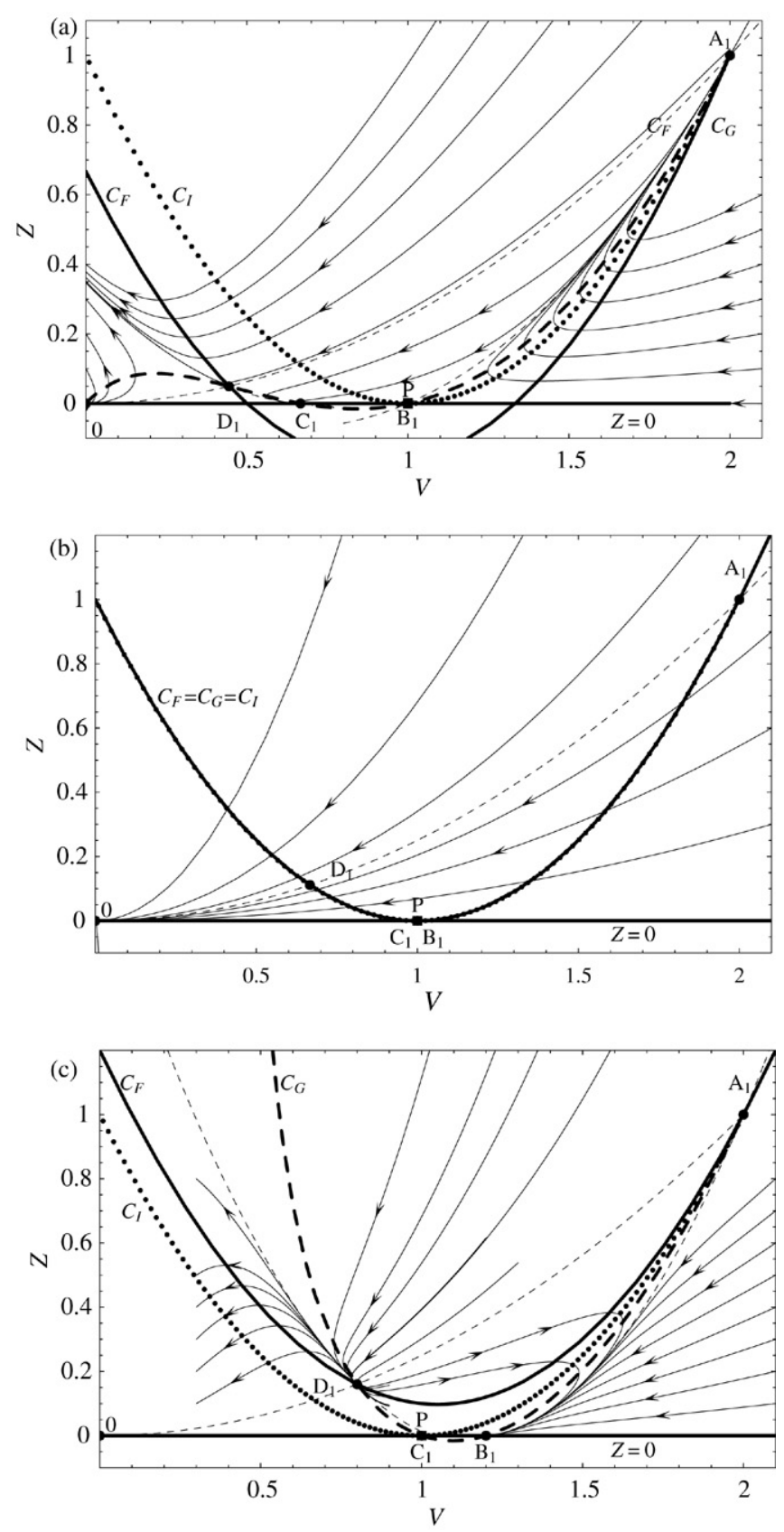

Fig. 7. Phase portraits for $\gamma=1$, (a) $\delta=3 / 2(n=5 / 2)$, (b) $\delta=1(n=1)$, and (c) $\delta=5 / 6(n=1 / 2)$. The key is the same as for Fig. 6 .

Appendix D for the mathematical proofs. In the latter case $(\alpha<1)$, we might be eager to use the same method to construct a solution, but in that case, this solution is nonphysical. Indeed, the integral path coming from the source $\mathrm{S}^{\prime}$ intersects $C_{I}$ at $\mathrm{E}^{\prime}$ (see Fig. 8) on the left of $\mathrm{P}$; the path $\mathrm{E}^{\prime} \rightarrow \mathrm{P}$ on $C_{I}$ points in the direction of decreasing $\xi$, which is physically inconsistent.

For $\delta<1$, we systematically meet the same difficulty as earlier with $\delta=1$ and $\alpha<1$. Indeed, the only integral path reaching $\mathrm{P}$ is the separatrix $S_{\mathrm{CD}}$, as shown in Fig. 7(c). Since any path on this separatrix pointing toward $\mathrm{P}$ is associated with decreasing $\xi$, we cannot build physical solutions.

More interesting, for $\delta>1$ and supercritical flow conditions at the source $(\alpha>1)$, we can construct similarity solutions

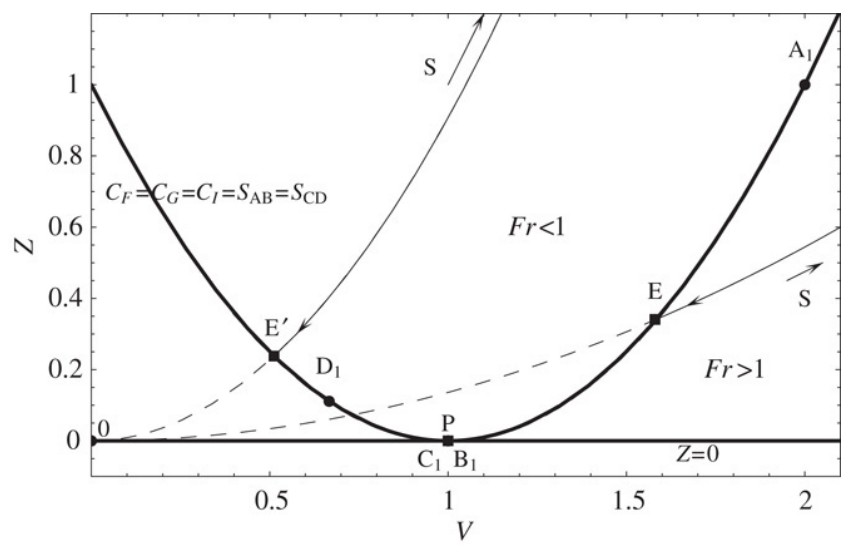

Fig. 8. Phase portraits for $\delta=1(n=1)$. The bold line represents the coalesced curves $C_{I}, C_{G}, C_{F}, S_{\mathrm{AB}}$, and $S_{\mathrm{CD}}$. The thin lines represent two pieces of integral path between the source point and the point of intersection with $C_{I}$, referred to as $\mathrm{E}(\alpha>1)$ and $\mathrm{E}^{\prime}(\alpha<1)$.

with discontinuities as follows. As earlier for $\delta=1$, we start from the source point and follow an integral path. Since the integral paths cross the critical curve $C_{I}$, a shock occurs. In the phase plane, the point where this shock occurs is called point E. As shown in Fig. 9(a), from this point, we can draw two shock curves corresponding to the shock conditions (11) and (12) [see also Eqs. (A.7) and (A.8) and further comments in Appendix A], but a single one is physical: the dashed line pointing to the left is associated with an energy loss, as expected in a hydraulic jump. This shock curve maps point $\mathrm{E}$ onto another point on separatrix $S_{\mathrm{AB}}$, which is referred to as point $\mathrm{E}^{\prime}$. Between $\mathrm{E}^{\prime}$ and $\mathrm{P}$, the integral path follows the separatrix $S_{\mathrm{AB}}$. This construction does not make sense when the flow is subcritical at the source $(\alpha<1)$ since no jump occurs. Fig. 9(a) and (b) show the flow depth and velocity profiles as a function of the similarity variable $\xi$ for the particular case $n=5 / 2(\delta=3 / 2), \alpha=3, \beta=1$, and $\gamma=1$ (dashed lines).

It is worth noting that for $\delta>1$ and $\alpha>1$, we may also hypothesize that within the body, there is shear in the vertical direction (i.e., $\gamma>1$ ), whereas within the tip region, the Boussinesq coefficient drops to unity since the hydraulic jump leads to profoundly altering the velocity profile [4]. With these assumptions, we can construct solutions where the flow conditions are supercritical at the source and the velocity profile is sheared. A typical example is reported in Fig. 9 (dashed line) for the particular case $\gamma=77 / 72$. As seen in this figure by comparing the dashed and solid curves, small changes in $\gamma$ lead to noticeable changes in the flow depth and velocity profiles.

\subsection{Summary}

A thorough analysis of the phase portraits has shown that unlike in the Boussinesq regime investigated by Grundy and Rottman [26] and Gratton and Vigo [25], the shallow-water equations for non-Boussinesq regimes do not systematically admit similarity solutions. For these similarity solutions to exist, the front point $\mathrm{P}$ must be a singular point of the phase portrait. This condition is met solely when $\gamma=1$ (at least in the immediate vicinity of the front) and $\delta \geq 1$, that is, there 

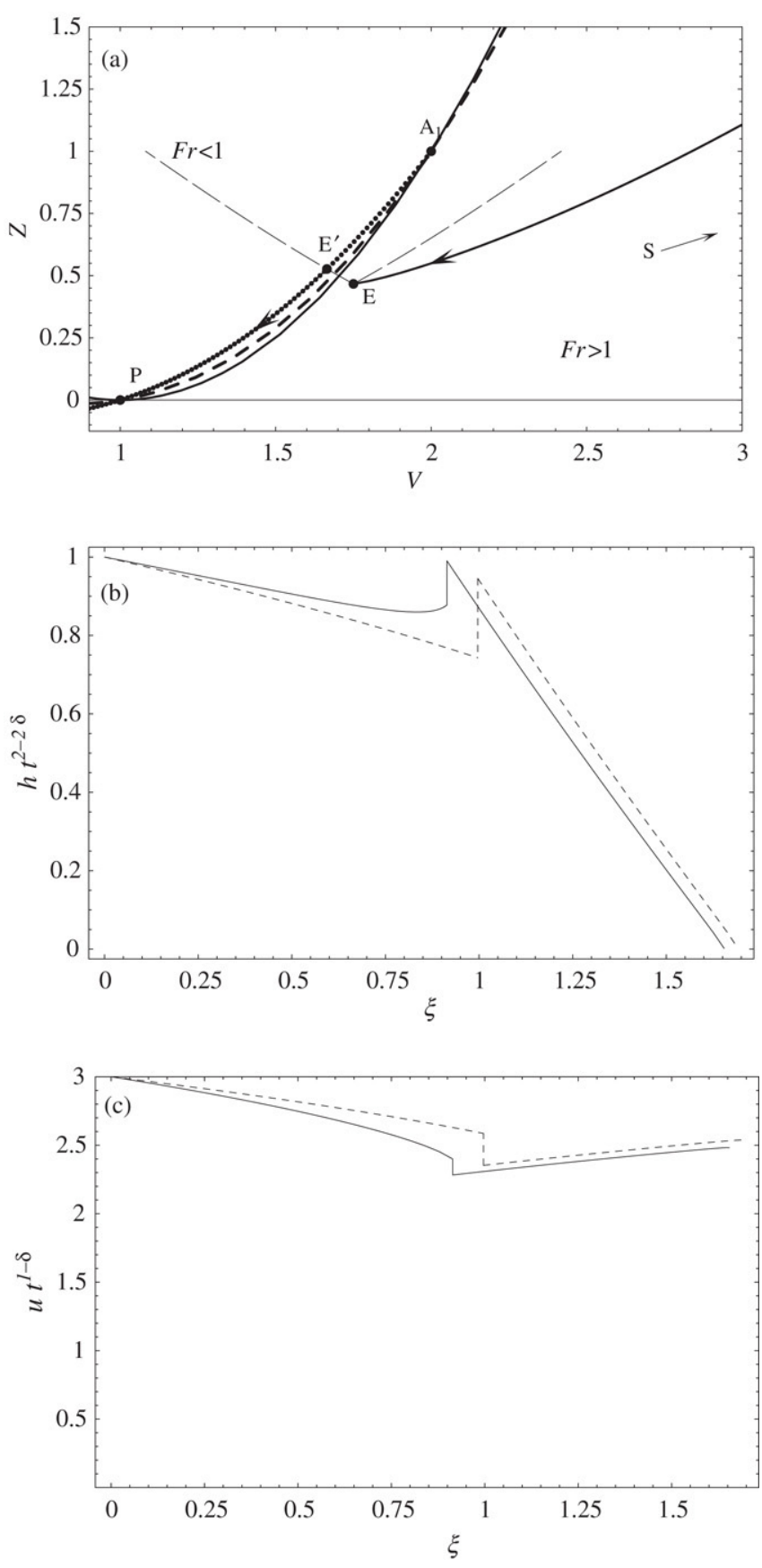

Fig. 9. (a) Phase plane in the vicinity of $\mathrm{A}_{1}$ : the specific curves $C_{I}$ (solid line), $C_{F}$ (dashed line) together with the separatrix $S_{\mathrm{AB}}$ (dotted line) are reported. The singular point $A_{1}$ is plotted together with the front point $P$. The long dashed lines represent the shock curves given by Eqs. (A.7) and (A.8) and emanating from point E. (b) Flow depth variation with $\xi$ : the solid line represents the solution obtained when $\gamma=1$ throughout the flow, while the dashed curve stands for the solution derived when $\gamma=77 / 72$ within the body and $\gamma=1$ within the head. (c) Velocity variation with $\xi$ : the same key as for (b). Computations made for $n=5 / 2, \alpha=3, \beta=1\left(A=\alpha \beta^{3 / 2} / n=1.2\right)$.

must be no shear in the velocity profile within the tip region and the total surge volume must increase vigorously with time: $\mathcal{V} \propto t^{n}$, with $n=3 \delta-2 \geq 1$. Furthermore, the flow must be supercritical at the source, which implies the existence of discontinuities in the flow depth profiles (when $\delta>1$ ) or in the
Table 2

Summary of the solutions depending on the Froude number $(\alpha)$ at the source, the Boussinesq coefficient $\gamma$, and the exponent $\delta$

\begin{tabular}{llll}
\hline$\delta$ & $\gamma$ & $\alpha \geq 1$ & $\alpha<1$ \\
\hline$\delta>1$ & $\gamma=1$ & $\begin{array}{l}\text { Discontinuous solution (jump) } \\
\text { Discontinuous solution (jump + change } \\
\text { in the velocity profile) }\end{array}$ & $\begin{array}{l}\text { No solution } \\
\text { No solution }\end{array}$ \\
\hline$\delta=1$ & $\gamma=1$ & $\begin{array}{l}\text { Continuous solution with discontinuous } \\
\text { gradient (Ritter-like solutions) }\end{array}$ & No solution \\
& $\gamma>1$ & $\begin{array}{l}\text { Discontinuous solution (jump + change } \\
\text { in the velocity profile) }\end{array}$ & No solution \\
\hline$\delta<1$ & $\gamma \geq 1$ & No solution & No solution
\end{tabular}

depth-gradient profiles (when $\delta=1$ ). Table 2 summarizes the conditions of existence depending on the values of $\alpha$ and $\beta$.

\section{Body dynamics}

To further investigate the behavior of non-Boussinesq gravity currents, the next step is to consider the Navier-Stokes equations and to rescale them to obtain simplified governing equations. Essentially, two approximations help simplify the problem. First, when working at high Reynolds numbers $R e=$ $U_{*} H_{*} / v$ with $v$ the kinematic viscosity, the viscous effects become negligible compared to inertia terms. Second, after introducing the aspect ratio $\epsilon=H_{*} / L_{*}$ and assuming that the flow is shallow $\epsilon \ll 1$, we can get rid of a number of terms in the Navier-Stokes equations by keeping only the terms whose order of magnitude is $O\left(\epsilon^{0}\right)$ (see Appendix E). Obviously, such an approximation no longer holds in the vicinity of the front because of the curvature of the free surface $(\epsilon \sim 1)$. This means that we have to consider the body and the front separately. In this section, we focus our attention on the body, while in Section 6, we will tackle the front dynamics. Fig. 10 shows the decomposition of the flow domain into two parts. We will show that the body solution is valid between the source and a point referred to as $M\left(0 \leq x \leq x_{m}\right)$, while the head solution is valid for $x \leq x_{a}$. Patching the two solutions is difficult because of significant changes in vorticity between the body (nearly irrotational flow) and the head (rotational flow). Our patching strategy is based on mass balance conservation arguments (see Section 7.2).

\subsection{Local governing equations in the high-Reynolds-number limit}

For the body of shallow flows in the high-Reynolds-number limit, we end up with the same governing equations as were obtained for inviscid shallow waves [10]:

$$
\begin{aligned}
& \frac{\partial u}{\partial x}+\frac{\partial v}{\partial y}=0, \\
& \frac{\partial u}{\partial t}+u \frac{\partial u}{\partial x}+v \frac{\partial u}{\partial y}=-\frac{\partial h}{\partial x},
\end{aligned}
$$

where $u(x, y, t)$ and $v(x, y, t)$ denote the horizontal and vertical components of the velocity field. These equations 


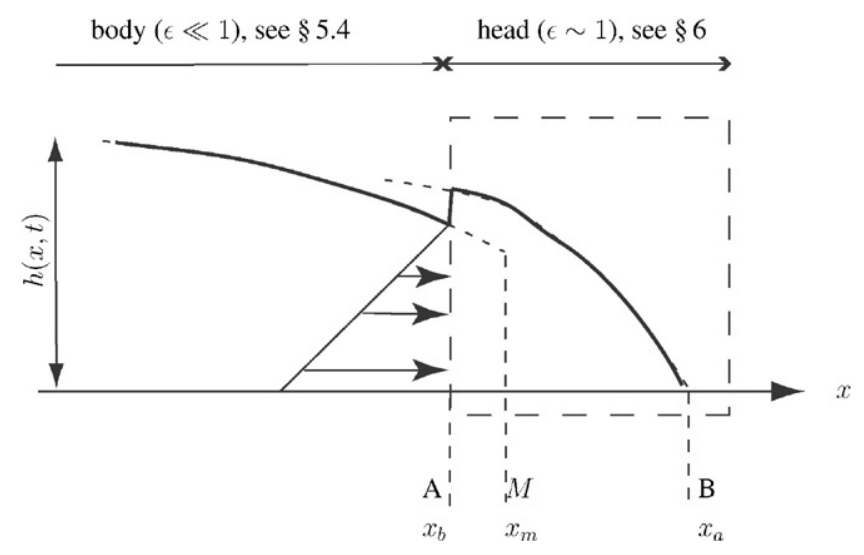

Fig. 10. Decomposition of the flow domain into a body and head. The dashed box represents the integral volume used to compute the characteristics of the nose.

are an approximation of the Euler equations when pressure is hydrostatic, i.e., the vertical component of the momentum balance equation (E.3) has been integrated to yield $p=h-y$. These equations are usually referred to as the shallow-wave equations.

The boundary conditions are the following:

at $x=0, h \bar{u}=n A t^{n-1}, h(0, t)=\beta t^{m} \quad$ and

$\operatorname{Fr}=\frac{\bar{u}}{\sqrt{h}}=\alpha$,

at $y=0, v=0$,

at $y=h, v=D h / D t$,

with $m=2(n-1) / 3, D / D t=\partial_{t}+u(x, h, t) \partial_{x}$, and $\bar{u}(x, t)=$ $\int_{0}^{h} u(x, y, t) \mathrm{d} y$. Following Blythe et al. [10], we will use the change of variables below:

$z=\frac{y}{h} \quad$ and $\quad w=\frac{D z}{D t}=\frac{1}{h}\left(v-z\left(h_{t}+u h_{x}\right)\right)$,

which makes it possible to simplify the formulation of the boundary conditions and introduce the flow depth solely in the governing equations. We finally obtain [10]

$h_{t}+u h_{x}+h\left(u_{x}+w_{z}\right)=0$,

$u_{t}+u u_{x}+w u_{z}+h_{x}=0$,

with $w=0$ at $z=0$ and $z=1$. This change of variables is relevant only for the body since it would become singular for the front point.

\subsection{Similarity solutions}

Similarity transformations of different kinds have been proposed to gain insight into the behavior of the solutions to Eqs. (25) and (26) [10,24,42,43,55], but none of these transformations provide similarity forms that are consistent with the treatment given in Section 4 for the shallow-water equations.

The first idea that comes up to mind is to assume that the velocity profiles must be self-similar, which allows us to decouple the dependence on $x$ and $z$, e.g., by posing $u=$ $t^{a} U(\xi) A(z), v=t^{b} W(\xi) B(z), h=t^{c} H(\xi)$, and $\xi=x / t^{d}$. Substituting these forms into the mass and momentum balance equations (25) and (26) and taking the boundary conditions (21) into account leads to: $a=\delta-1, b=-1, c=2(\delta-1)$, $d=\delta$. By doing this, we also obtain a set of two equations for $U, W, A$, and $B$. Solving these equations leads to finding that $U(z)=1$ and $B(z)=z$, which is nothing but the velocity profile that was assumed in the derivation of the shallow-water equations when the Boussinesq coefficient was set to unity. In short, with these similarity forms, we will learn nothing more than we already know.

This prompts us to seek more complex similarity forms, but still keeping in mind that they must be consistent with the similarity forms (13) and (14) used for the shallow-water equations. Taking a closer look at the phase portrait drawn for the shallow-water equations provides interesting hints. In Section 4, dominant-balance analysis demonstrated that close to the source, $V \propto \sqrt{Z}$; numerical simulations showed that this approximation held over a large part of the integral paths, but broke in the immediate vicinity of the front. Since $V \propto \sqrt{Z}$ is equivalent to $\bar{u} \propto \sqrt{h}$, this impels us to impose

$u(x, z, t)=\sqrt{h(x, t)} U(z)$,

$w(x, z, t)=\frac{1}{\sqrt{h(x, t)}} \frac{\partial h(x, t)}{\partial x} W(z)$,

$h(x, t)=t^{2(\delta-1)} H(\xi)$,

$\xi=\frac{x}{t^{\delta}}$.

Since the shallowness approximation breaks within the tip region, we cannot use the governing equations (25) and (26) to compute the flow features over the whole domain; the similarity forms (27)-(30) hold only for the body. Note that the structure of the similarity expression (28) was dictated by the continuity equation (19). The structure of the horizontal velocity expression (27) shows that $u$ is a function of $x$ and $t$ through its dependence on the flow depth $h(x, t)$; this means that on average, the fluid velocity adapts instantaneously to any change in the flow depth.

Another implication is that integrating the continuity equation (19) leads to

$h_{t}+c(h) h_{x}=0$,

or equivalently

$\bar{u}_{t}+c(h(\bar{u})) \bar{u}_{x}=0$

with $c(h)=\bar{u}+h \bar{u}^{\prime}=3 \bar{u} / 2$, where $\bar{u}^{\prime}$ denotes the $h$ derivative of the mean velocity. This shows that the solution sought belongs to the family of 'simple' kinematic waves (see [40] for an introduction to simple kinematic waves).

We substitute the similarity forms (27)-(30) into the governing equations (25) and (26) and obtain

$4(\delta-1) H^{3 / 2}+\left(3 U H+2 W^{\prime} H-2 \delta \xi \sqrt{H}\right) H^{\prime}=0$,

$2 U H(\delta-1)+\left(\sqrt{H}\left(U^{2}+2\right)-U \delta \xi+2 W \sqrt{H} U^{\prime}\right) H^{\prime}=0$, 
which can be transformed into expressions that are easier to interpret:

$2(\delta-1) H+\frac{1}{2} \sqrt{H} H^{\prime}\left(3 U+2 W^{\prime}\right)-\delta \xi H^{\prime}=0$,

$U^{2}+U \frac{2 H(\delta-1)-H^{\prime} \delta \xi}{\sqrt{H} H^{\prime}}+2 W U^{\prime}+2=0$.

Since $U$ and $W$ are functions of $z$ alone while $H$ depends solely on $\xi$, we derive the following conditions:

$3 U+2 W^{\prime}=2 c_{1} \quad$ and $\quad \frac{2 H(\delta-1)-H^{\prime} \delta \xi}{\sqrt{H} H^{\prime}}=c_{2}$,

where $c_{1}$ and $c_{2}$ are two constants to be determined. Comparing these conditions with Eq. (32) leads to $c_{2}=-c_{1}$. Eliminating $W$ from Eq. (33) by differentiating this equation with respect to $z$, we deduce that the function $U$ satisfies the second-order differential equation

$U^{\prime \prime}\left(2+U\left(U-c_{1}\right)\right)+U^{\prime 2}\left(U-c_{1}\right)=0$,

while the function $W$ is a solution of

$2 W^{\prime}=2 c_{1}-3 U$,

and the flow depth $H$ satisfies

$2(\delta-1) H+H^{\prime}\left(c_{1} \sqrt{H}-\delta \xi\right)=0$.

\subsection{Velocity profiles}

Eq. (34) can be transformed into a linear second-order differential equation by using an inverse transformation

$\left(U-u_{1}\right)\left(U-u_{2}\right) \frac{\mathrm{d}^{2} z}{\mathrm{~d} U^{2}}+\left(c_{1}-U\right) \frac{\mathrm{d} z}{\mathrm{~d} U}=0$,

where $u_{1}=\left(c_{1}-\sqrt{c_{1}^{2}-8}\right) / 2$ and $u_{2}=\left(c_{1}+\sqrt{c_{1}^{2}-8}\right) / 2$ are the two roots of $U\left(c_{1}-U\right)-2=0$. For the roots to be real, the parameter $c_{1}$ must satisfy $c_{1}>2 \sqrt{2}$. When $c_{1}<2 \sqrt{2}$, a nonphysical behavior (i.e., negative streamwise velocity) is obtained.

Using the change in variable $\sigma=\left(U-u_{1}\right) /\left(u_{2}-u_{1}\right)$, one obtains

$\sigma(1-\sigma) \frac{\mathrm{d}^{2} z}{\mathrm{~d} \sigma^{2}}+\left(\sigma-\sigma_{1}\right) \frac{\mathrm{d} z}{\mathrm{~d} \sigma}=0$,

with $\sigma_{1}=2\left(2-u_{1}^{2}\right)^{-1}$. The solution to this differential equation can be expressed in terms of the incomplete Beta function [1]:

$z=\frac{\operatorname{Be}\left(\sigma, 1+\sigma_{1}, 2-\sigma_{1}\right)}{\operatorname{Be}\left(1,1+\sigma_{1}, 2-\sigma_{1}\right)}$.

We now determine the constant $c_{1}$ (and hence $u_{1}, u_{2}$, and $\sigma_{1}$ ) by computing the flow depth averaged horizontal velocity $\bar{u}$. Using the relation $\operatorname{Be}(p+1, q)=\frac{p}{p+q} \operatorname{Be}(p, q)$ holding for any pair of reals $(p, q)$, we find

$\int_{0}^{1} U(z) \mathrm{d} z=\left(u_{2}-u_{1}\right) \frac{1+\sigma_{1}}{3}+u_{1}=\frac{2}{3} c_{1}$,
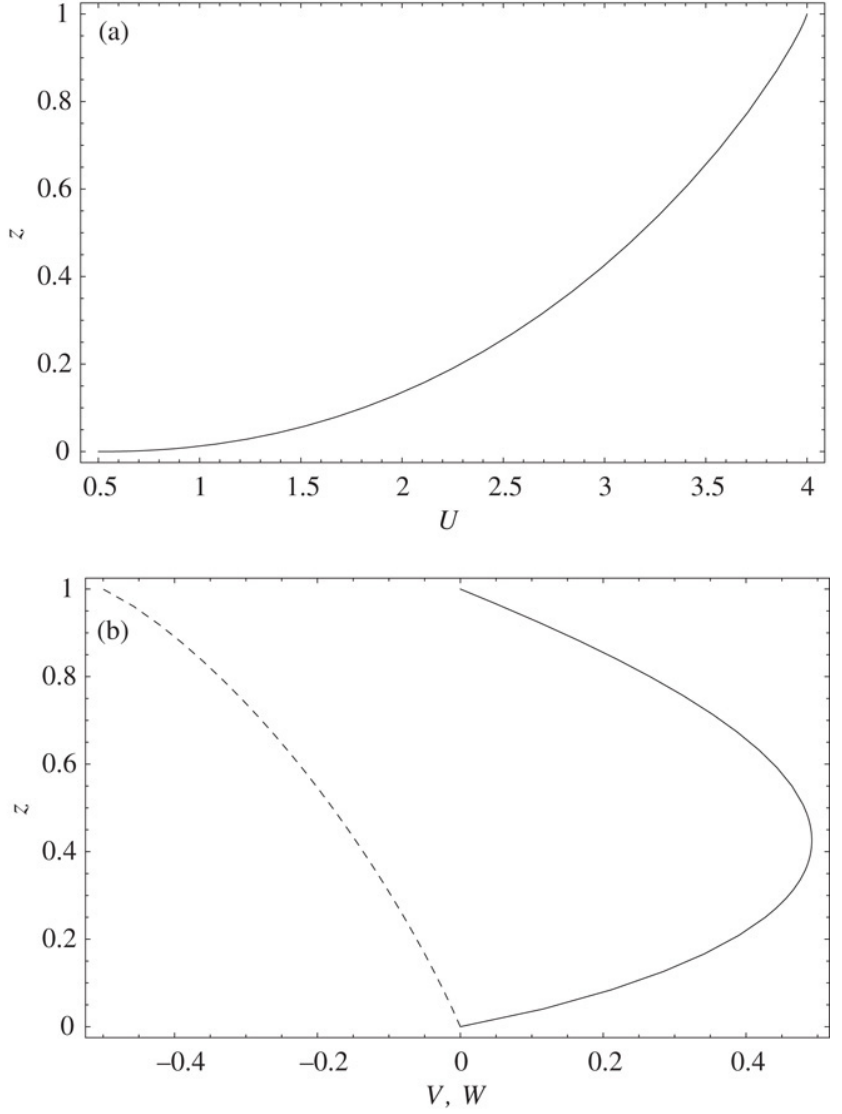

Fig. 11. Velocity profiles: (a) horizontal velocity $U$; (b) scaled vertical velocity $W$ (solid line) and physical vertical velocity $V$ (dashed line). Computations made in the particular case $\alpha=3$.

or equivalently

$\bar{u}(x, t)=\frac{2}{3} c_{1} \sqrt{h(\xi)}$.

The boundary condition (21) implies that $c_{1}=3 \alpha / 2$. The condition for existence of real solutions is then that the Froude number at the source satisfies

$\alpha>\frac{4}{3} \sqrt{2} \approx 1.88$

The function $W(z)$ is directly derived from Eq. (33)

$W=-\frac{2+U(U-3 \alpha / 2)}{2 U^{\prime}}$.

Fig. 11 shows the velocity profiles $U$ and $W$ for $\alpha=3$ and $n=5 / 2$. We can also plot the variations in the vertical velocity. Indeed, using the change of variable (24) backwards together with Eq. (25), we can express the dimensionless vertical component of the velocity as

$v(x, y, t)=h\left(w-z u_{x}-z w_{z}\right)=h^{1 / 2}(\xi) h_{x}(\xi) V(z)$,

with $V(z)=W(z)-z U(z) / 2-z W^{\prime}(z)$. Note that the streamwise component of the bottom velocity is low, but nonzero: the slipping velocity is $U(0)=u_{1}$. In Fig. 11, it is seen that the streamwise component of the velocity has a profile close to a parabolic shape. The vertical component of the 
velocity $(v)$ is negative because of the curvature of the free surface.

\subsection{Flow depth profile}

Similarly, we can derive the flow depth profile analytically. By setting $H=\xi^{2} \Omega^{2}$ in Eq. (36), we obtain a homogeneous (i.e., separable) first-order differential equation

$\xi \frac{\mathrm{d} \Omega}{\mathrm{d} \xi}=\Omega \frac{3 \alpha \Omega-2}{3 \alpha \Omega-2 \delta}$,

which when integrated provides

$$
\ln \left(c_{3} \xi\right)=\ln \left|\frac{(3 \alpha \Omega-2)^{\delta-1}}{\Omega^{\delta}}\right|,
$$

with $c_{3}>0$ a constant of integration. When $n>1$, this equation can also be put in the implicit form

$\xi=-\frac{1}{3} c_{3}^{1 /(\delta-1)} H^{s}+\frac{3 \alpha}{2} H^{1 / 2}$,

with $s=\delta /(2 \delta-2)=(2+n) /(2 n-2)>1 / 2$ for $n>1$. For $\xi=0$, this equation provides the constant of integration as a function of the initial flow depth

$c_{3}=\frac{1}{\sqrt{\beta}}\left(\frac{9 \alpha}{2}\right)^{\delta-1}$.

Taking a closer look at the $\xi$ variation with $H$ [see Eq. (42)] shows that the function $\xi(H)$ is a bell-shaped curve, which takes it maximum at a point $\mathrm{M}$ (see Fig. 12):

$\xi_{m}=\frac{3}{2} \alpha \sqrt{\beta}(\delta-1)^{\delta-1} \delta^{-\delta} \quad$ and $\quad H_{m}=\beta\left(\frac{\delta-1}{\delta}\right)^{2(\delta-1)}$.

It is easily seen from Eq. (42) that the flow depth vanishes at $\xi=0$ only. When $n=1(\delta=1)$, Eq. (36) reduces to $H^{\prime}\left(c_{1} \sqrt{H}-\xi\right)=0$, which provides two solutions: $H=\beta$ or $H=\left(\xi / c_{1}\right)^{2}$. Since the latter does not satisfy the downstream boundary condition, the solution is $H=\beta$, which is consistent with what we can anticipate for a steady inflow.

\section{Front dynamics}

Close to the front, the shallowness approximation $(\epsilon \ll 1)$ is no longer valid because of the significant increase in the free-surface curvature. In order to compute the main features of the front, we will first use an integral method similar to the treatment proposed by Whitham [65] in his calculation of the drag-resistance effect on the front propagation of a wave induced by a dam break. Then we will examine the full Euler equations to further characterize the front behavior and determine its structure.

\subsection{Bulk analysis of the head}

The immediate vicinity of the front is a special region: the wave velocity and the material velocity should coincide, which

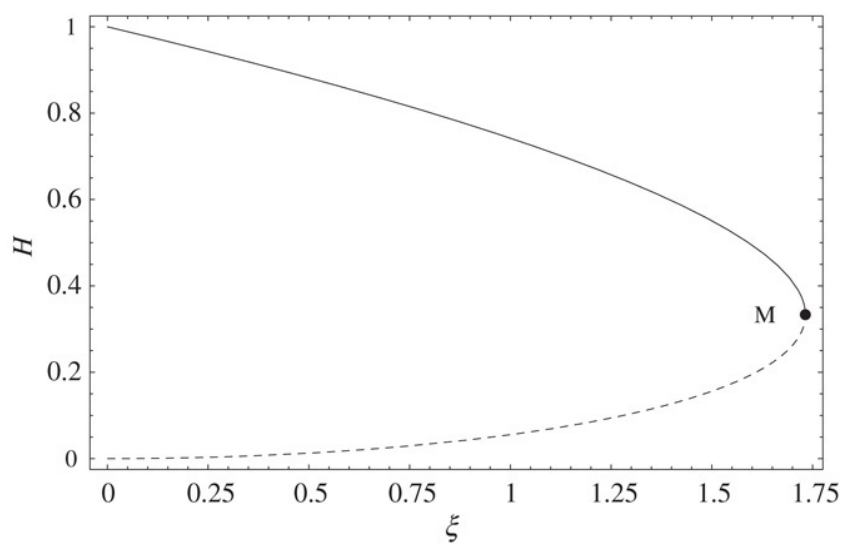

Fig. 12. Variation of $\xi(H)$ : the solid line represents the physical branch, while the dashed line represents the nonphysical branch. Computations made for $\alpha=3, \delta=3 / 2$, and $\beta=1$.

imposes that the material velocity is $\dot{x}_{a}$ close to the front, where the dot refers to the time derivative. In a first approximation, we shall consider that, within the tip region, the velocity is uniform and equal to the front velocity, which makes it possible to derive the governing equations of the front by using an integral representation of the mass and momentum balance equations.

For this purpose, let us consider that the head is the flow domain comprised between points A and B, as shown in Fig. 10: point A of abscissa $x_{a}$ represents the actual front location. Point B of abscissa $x_{b}$ corresponds to the junction between the head and the body; for $x \leq x_{b}$, the similarity solution found earlier holds (see Section 5). We are now writing up the governing equations for the integral volume between $x_{a}$ and $x_{b}$. Since the upstream border of the integral volume moves at a velocity $\dot{x}_{b}$, the mass flux through this moving boundary is $\int_{0}^{h_{b}}\left(u_{b}-\dot{x}_{b}\right) \mathrm{d} y$; the mass balance can then be expressed in a dimensionless form as

$\frac{\mathrm{d} M}{\mathrm{~d} t}=\int_{0}^{h_{b}}\left(u_{b}-\dot{x}_{b}\right) \mathrm{d} y=h_{b}\left(\bar{u}_{b}-\dot{x}_{b}\right)$,

where $M$ is the mass of fluid contained in the tip region between $x_{b}$ and $x_{a}, h_{b}$ is the flow depth at the junction point $\mathrm{B}$, and $\bar{u}_{b}$ is the flow depth averaged velocity at $\mathrm{B}$. The integral momentum balance equation can be expressed as

$\frac{\mathrm{d} P}{\mathrm{~d} t}=h_{b} \bar{u}_{b}\left(\bar{u}_{b}-\dot{x}_{b}\right)+\frac{1}{2} h_{b}^{2}$,

where $P$ is the bulk momentum. The first term on the right-hand side represents the momentum flux across the moving boundary at $\mathrm{B}$, the second term is the pressure force exerted by the body on the volume. In the momentum balance equation, we still neglect viscous dissipation.

We are now transforming the momentum equation into a governing equation for $\dot{x}_{a}$. To that end, we must express all variables in terms of $\dot{x}_{a}$. Since the velocity within the tip is very close to the wave velocity, we use the approximations

$\bar{u}_{b}=\alpha \sqrt{H_{b} t^{(2 \delta-2)}} \approx \dot{x}_{a}$ and $P \approx M \dot{x}_{a}$, 
with $H_{b}=H\left(\xi_{b}\right)$. We then derive the expression of $H_{b}$ as a function of $\dot{x}_{a}: H_{b}=t^{-(2 \delta-2)}\left(\dot{x}_{a} / \alpha\right)^{2}$. Furthermore, from Eq. (42), we have $\xi_{b}=-c_{3}^{1 /(\delta-1)} H_{b}^{s} / 3+3 \alpha H_{b}^{1 / 2} / 2$.

Integrating the mass balance equation leads to

$M=H_{b}\left(\alpha \sqrt{H_{b}}-\delta \xi_{b}\right) \frac{t^{n}}{n}$,

and we finally deduce

$\ddot{x}_{a}\left(\left(1-\frac{3}{2} \delta\right) t \frac{\dot{x}_{a}}{n}+\delta \frac{c_{3}^{1 /(\delta-1)}}{3 n}\left(\frac{\dot{x}_{a}}{\alpha}\right)^{2 s}\right)=\frac{1}{2}\left(\frac{\dot{x}_{a}}{\alpha}\right)^{2}$.

Looking for a long-term asymptotic approximation, we impose $x_{a}=\mathcal{A} t^{p}$. Injecting this ansatz in the governing equation (45), we find $p=\delta$ and

$\xi_{a}=\mathcal{A}=\frac{3^{-2 n / 3} \alpha \sqrt{\beta}}{n+2}\left(\frac{9(n+2)(n-1) \alpha^{2}}{n\left(3^{4+\frac{5}{n-1}}(n-1) \alpha^{2}+3^{\frac{5 n}{n-1}}\right)}\right)^{\frac{1-n}{3}}$.

Using Eq. (42), we then deduce the abscissa of the transition point B:

$\xi_{b}=\frac{3 \delta}{2} \xi_{a}-\frac{3}{2} \alpha \beta^{-3 /(2 n-2)}\left(\frac{\delta}{\alpha} \xi_{a}\right)^{2 s}$.

Numerical solution shows that the asymptotic solution $x_{a}=$ $\mathcal{A} t^{p}$ is a fairly good approximation of the solution at short and long times.

Several comments can be made. The front position A has the same asymptotic time dependence as the point $\mathrm{M}$, as expected. We also note that the actual front abscissa $x_{a}$ is not too far from the position $x_{m}$ computed in Section 5.4. For instance, taking $n=5 / 2$ (i.e., $\delta=3 / 2$ ), $\alpha=3$, and $\beta=1$ gives $\xi_{a} \approx 1.648$, $\xi_{b} \approx 1.190$, and $\xi_{m} / \xi_{a}=1.05$. Fig. 13 reports the variations of the similarity variables $\xi_{a}$ and $\xi_{m}$ as functions of the Froude number $\alpha$ in the particular case $n=5 / 2$. The ratio $\xi_{m} / \xi_{a}$ is independent of the aperture $\beta$ and depends only on the Froude number $\alpha$ and the growth rate $n$. We observed that the variables $\xi_{a}$ and $\xi_{m}$ are very close, especially at low $\alpha$ values. We failed to find any physical reason why this should be so. The head length $x_{a}-x_{b}$ is fairly large at low Froude numbers, which means that the front cannot be reduced to a thin region. However, its length progressively decreases with increasing Froude numbers, which shows that the front is much steeper at high Froude numbers.

The only assumption made in arriving at this result has been stating that the velocity is fairly constant within the tip, an assumption that it is not correct, as we will show below, but provides a satisfactory approximation at long times. On the whole, the front dynamics is entirely controlled by a balance between the pressure gradient and the momentum variation inside the front. As is expected for supercritical flows, the front exerts no control on the body.

\subsection{Refined analysis of the front structure}

We now return to the local Navier-Stokes equations (E.2) and (E.3) made dimensionless, but now with $\epsilon \sim 1$ because

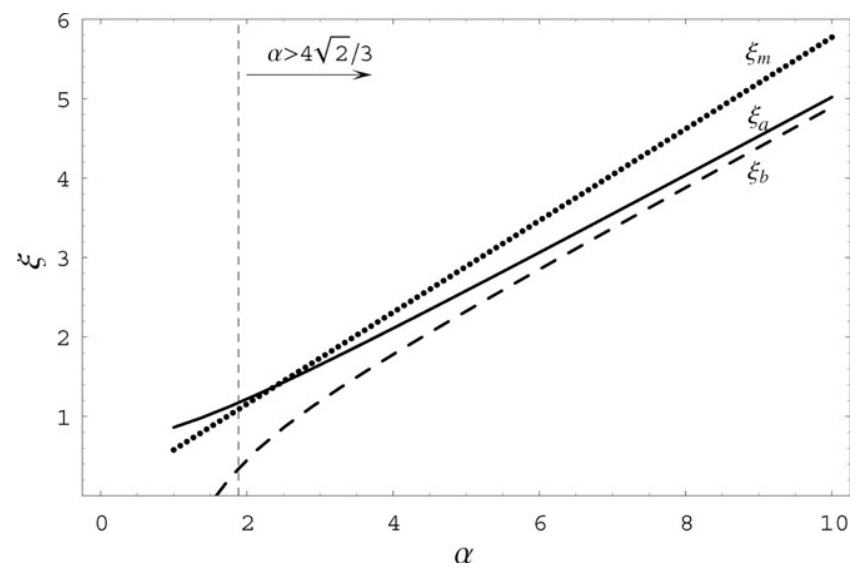

Fig. 13. Variation of the front position $\xi_{a}$ (solid line) and $\xi_{b}$ (dashed line) as a function of the Froude number $\alpha$ (computations made with $\beta=1$ and $n=5 / 2$ ). We have also reported the variation $\xi_{m}$ (dotted line). $\xi_{a}, \xi_{b}$, and $\xi_{m}$ are given by Eqs. (46), (47) and (43), respectively.

of the significant variation in the flow depth within the leading edge. Introducing the stream function $\psi\left(u=\psi_{y}\right.$ and $v=$ $-\psi_{x}$ ) and taking the curl of the equations to get rid of the pressure gradient provides the vorticity equation, where $\omega=$ $-\Delta \psi$ is the vorticity

$\frac{\partial}{\partial t} \Delta \psi+\left(\frac{\partial \psi}{\partial y} \frac{\partial}{\partial x}-\frac{\partial \psi}{\partial x} \frac{\partial}{\partial y}\right) \Delta \psi=0$.

This equation remains invariant under the transformation $(x, y, t, \psi) \rightarrow\left(x+x_{a}(t), y, t, \psi+y \dot{x}_{a}\right)$, which represents a nonuniformly accelerated translation [12]. Let us consider a Cartesian frame of reference fixed with respect to the leading edge, i.e. positioned at $\mathrm{A}$ and moving at the velocity $\dot{x}_{a}=$ $\delta A t^{\delta-1}$ with respect to a fixed frame (attached to the bed).

For $\delta<2(n<4)$, this frame is nonuniformly accelerated, but the acceleration term is proportional to $\ddot{x}_{a} \propto t^{\delta-2}$, which implies that at long times, the acceleration effects are negligible and we can consider the frame as nearly Galilean. This prompts us to express the stream function in this frame as a time series: $\psi=\psi_{0}(x, y)+t^{-1} \psi_{1}(x, y)+\cdots$. Using this expansion provides a hierarchy of equations. At order $O\left(t^{0}\right)$, we have

$\left(\frac{\partial \psi_{0}}{\partial y} \frac{\partial}{\partial x}-\frac{\partial \psi_{0}}{\partial x} \frac{\partial}{\partial y}\right) \Delta \psi_{0}=0$.

At order $O\left(t^{1}\right)$, we have $\psi_{1, y}\left(\Delta \psi_{0}\right)_{x}+\psi_{0, y}\left(\Delta \psi_{1}\right)_{x}=0$ and $\psi_{1, x}\left(\Delta \psi_{0}\right)_{y}+\psi_{0, x}\left(\Delta \psi_{1}\right)_{t}=0$.

The boundary conditions are the following: at the bottom $v=-\psi_{0, x}(x, 0)=0$. At order $O\left(t^{0}\right)$, the free boundary is a stationary line that coincides with a streamline. Without loss of generality, we can set $\psi_{0}=0$ along the free surface. Moreover, if the free surface is a streamline, this implies that according to the Bernoulli theorem, the velocity is constant along this boundary since the pressure is constant at the free surface. The bottom is also a streamline since a particle is forced to have a trajectory parallel to the bed line; since this streamline and the streamline at the free surface intersect at the leading edge, they have the same value; hence we have $\psi_{0}=0$ along the bottom. In order to compute the vorticity $\Delta \psi_{0}$, we need to know the 
(a)

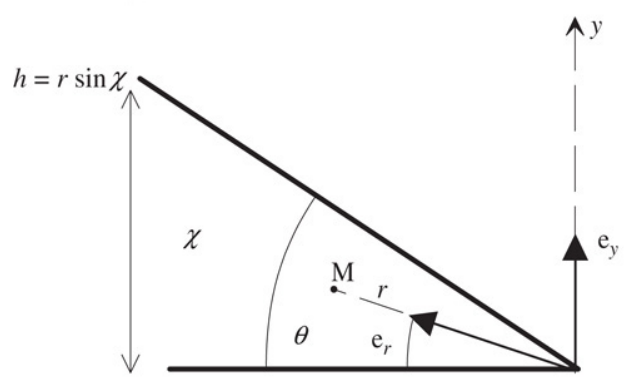

(b)

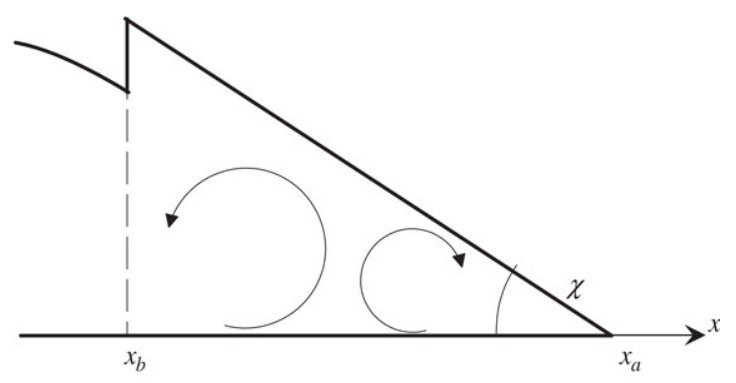

Fig. 14. (a) Notation used in the computation. (b) Vortices within the head.

vorticity in the far field. Using Eqs. (27)-(39), we find that

$$
\begin{aligned}
\omega= & \frac{\partial u}{\partial y}-\frac{\partial v}{\partial x}=\frac{\left(y\left(H^{\prime}\right)^{2} V^{\prime}\right) t^{-\delta-1}}{H^{3 / 2}}+\frac{U^{\prime} t^{1-\delta}}{\sqrt{H}} \\
& +\frac{\left(\left(H^{\prime}\right)^{2}+2 H H^{\prime \prime}\right)\left(-t^{\delta-3}\right) V}{2 \sqrt{H}},
\end{aligned}
$$

which implies that at order $O\left(t^{0}\right)$, the vorticity vanishes and the flow is irrotational in the body.

Since the flow is irrotational in the far field, we could first seek an irrotational solution to Eq. (49), but as shown in detail in Appendix F, this type of solution is hardly admissible from the physical point of view unless the front is associated with a mass sink. We are then looking for a rotational solution to Eq. (49). Using polar coordinates $(r, \theta)$ [see Fig. 14(a)], we assume that the variables are separable, i.e., the stream function can be expressed in the form: $\psi_{0}=f(r) g(\theta / \phi(r))$, where we have further assumed that the free boundary can be represented by a function $\theta=\phi(r)$. We also introduce the shorthand notation: $\Theta=\theta / \phi(r)$. We then have three functions to determine: $f(r), g(\Theta)$, and $\phi(r)$. At the free surface, three conditions must be satisfied: (i) the radial velocity $u=r^{-1} \psi_{0, \theta}=f(r) \phi^{-1}(r) g^{\prime}(\Theta)$ must be independent of $r$ since the pressure is constant at the free surface, (ii) the free boundary is a streamline $f(r) g(\Theta)=0$ for $\Theta=0$ or $\Theta=1$, (iii) the orthoradial component of the velocity vanishes $v=\psi_{0, r}=f^{\prime}(r) g(\Theta)-f(r) \phi^{-2}(r) \phi^{\prime}(r) g^{\prime}(\Theta)=0$. In addition, since the computations are made in the moving frame, the net mass flux across a surface $r=c s t$ spanning the tip must be zero, which implies that we must look for a piecewise continuous radial component of the velocity $u=g^{\prime}$ that is alternatively positive and negative such that $\int_{0}^{1} g^{\prime}(\Theta) \mathrm{d} \Theta=0$ at any $r$.

These equations admit at least one solution: we assume that there are two real constants $\chi$ and $c_{0}$ such that $\phi(r)=\chi$ and $f(r)=c_{0} r$. Because $g(\Theta)$ is assumed to be a nontrivial solution that vanishes on $\Theta=0$ and $\Theta=1$, we can add the following normalization constraint on the maximum value of $g$ on $[0,1]$ : there is at least one angle $\Theta_{m}$ such that $g\left(\Theta_{m}\right)=1$; the constant $c_{0}$ in $f(r)=c_{0} r$ must then be adjusted for this constraint to be satisfied. The free boundary must be a straight line making an angle $\chi$ with the bed line.

We impose $\Psi_{0}=c_{0} r g(\theta)$ (we no longer use the reduced variable $\Theta$ since $\phi$ is constant) and substitute it into the equivalent of Eq. (49) in polar coordinates. We deduce that $g$ must satisfy

$g^{\prime}\left(g+g^{\prime \prime}\right)+g\left(g^{\prime}+g^{\prime \prime \prime}\right)=0$,

which when integrated produces: $g g^{\prime \prime}+g^{2}+c_{1}=0$, where $c_{1}$ is a constant of integration. Multiplying this equation by $2 g^{\prime} / g$ and integrating it provides

$g^{\prime 2}+g^{2}+2 c_{1} \ln |g|=c_{2}$,

where $c_{2}$ is an integration constant. Using the normalization constraint on $g$, we find $c_{2}=1$. We failed to find an analytical solution to this first-order differential equation, but its behavior can be described by using asymptotic expressions and numerical approximations. Close to the boundary, $g \rightarrow 0$ implies that $g^{\prime} \sim \pm \sqrt{-2 c_{1} \ln |g|}$, implying that $c_{1}$ must be positive since $\ln |g|<0$. This differential equation can be integrated to provide

$|g| \sim \exp \left[-\operatorname{erf}^{(-1)}\left(1-\theta \sqrt{c_{1} / \pi}\right)\right]$,

for $\theta \rightarrow 0$, where $\operatorname{erf}^{(-1)}$ is the inverse of the error function. Close to the maximum, $g \rightarrow 1$ implies that $g^{\prime} \sim \pm \sqrt{1-g^{2}}$, i.e.,

$|g(\theta)| \sim \cos \left(\theta-\theta_{m}\right)$

for $\theta \rightarrow \theta_{m}$. Numerical integration reveals that

$|g(\theta)| \approx \sin ^{2 / 3}\left(\frac{\pi}{\chi} \theta\right)$ and $c_{1} \approx \frac{2 \pi^{2}}{3 \chi^{2}}-1$.

These numerical approximations are accurate to within a few per cent (less than 3\%). Fig. 15 shows a comparison between the numerical solution to Eq. (50) for $\chi=\pi / 6$ and the approximate function. Since the mass flux across any surface spanning the wedge must be zero, the derivative $g^{\prime}$ must be first negative for $0<\theta<\chi / 2$, then positive for $\chi / 2<\theta<\chi$; because of the symmetry imposed on the function $g^{\prime}$, there is a maximum of $g$ located at $\theta_{m}=\frac{1}{2} \chi$. The pressure can be determined by substituting the velocity components in the polar equivalent of the Euler equations and integrating these equations. We obtain

$p=-c_{1} c_{0}^{2} \ln r=-\frac{c_{1} c_{0}^{2}}{2} \ln \left(\left(x-x_{a}\right)^{2}+y^{2}\right)$, 


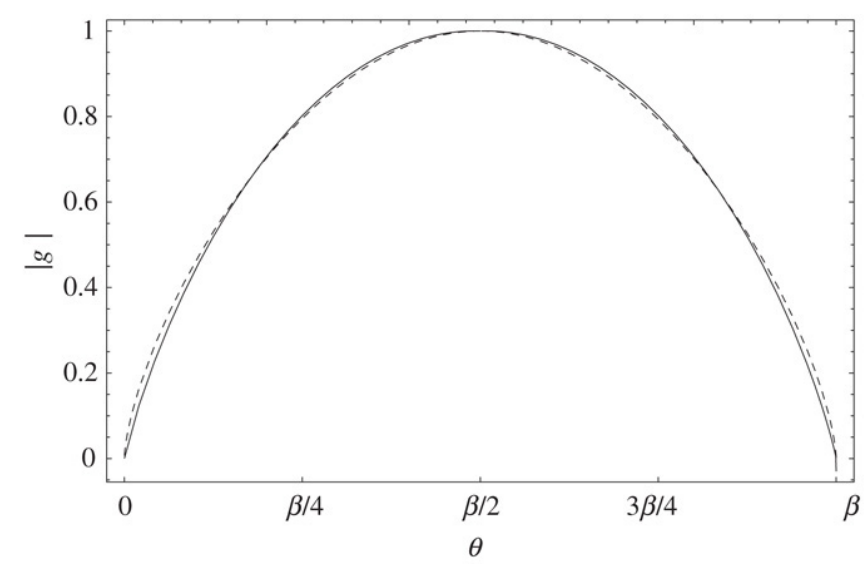

Fig. 15. Numerical solution to Eq. (50) for $\chi=\pi / 6$. The solid line represents the numerical solution, while the dashed line represents the numerical approximation equation (51).

showing that the pressure is independent of the angle $\theta$, is not hydrostatic within the tip region, and is singular at the leading edge; taking into account hydraulic resistance within the tip region will probably remove this singularity. Note that the pressure is not constant at the free boundary, but this does not violate the Bernoulli relation since the radial velocity becomes infinite along the streamline $\psi_{0}=0$ : the velocity components in the moving frame are $u=c_{0} g^{\prime}(\theta)$ and $v=-c_{0} g(\theta)$. This singularity can be removed by considering appropriate boundary conditions at the free surface: as suggested by McElwaine [46], the intrusion of a high-speed gravity current entails the motion of the ambient fluid, which means that there is a more complicated relation between the surge and its environment. As shown by McElwaine [46], the singularity in the $u$ and $p$ solutions drops out when the stream function has a highly pronounced dependence on $r$ : typically, we should impose $\psi_{0}=c_{0} r^{m} g(\theta)$ with $m>2$; the case $m=2$ corresponds to constant vorticity [23].

From the local components of the velocity in the moving frame, we deduce the streamwise velocity in the fixed Cartesian frame

$$
\begin{aligned}
u= & \dot{x}_{a}+c_{0} g^{\prime}\left(\tan ^{(-1)} z\right) \cos \left(\tan ^{(-1)} z\right) \\
& -c_{0} g\left(\tan ^{(-1)} z\right) \sin \left(\tan ^{(-1)} z\right)+O\left(t^{-1}\right),
\end{aligned}
$$

with $z=y /\left(x-x_{a}\right)$. This equation shows that the velocity profile is not uniform across the flow depth, but the vorticity contribution to $u$ becomes less important with increasing time and, at long times, the assumption of a uniform velocity profile becomes reliable.

In short, we have found a first-order solution to Eq. (48), showing that the free boundary is a straight line, at least close to the front. The flow is rotational with an infinite vorticity at the leading edge $(r=0)$ and along the boundaries $(g(\theta)=0)$

$\omega_{0}=-\nabla^{2} \psi_{0}=-c_{0} \frac{g^{\prime \prime}+g}{r}=\frac{c_{0}}{r} \frac{c_{1}}{g(\theta)}$,

due to the simplified boundary conditions considered here. For the flow to be irrotational far from the leading edge, we must contemplate a counter-clockwise vortex in the rear of the head, as depicted in Fig. 14(b). This vortex has no specific role other than creating a counter-reaction to the vorticity at the leading edge.

\section{Patching and comparison with the shallow-water equations}

\subsection{Patching}

An obvious shortcoming of the analysis pursued in Sections 5 and 6 is that the velocity profiles within the body and the head do not match, which means that a transitional slice between these regions associated with a significant increase in vorticity must exist. The same abrupt change in the flow behavior was predicted by the shallow-water equations in the form of a hydraulic jump. Although there is some analytical work for laminar viscous flows [29,63] and turbulent flows [11, 31], further analyzing the characteristics of the transitional slice here is beyond the scope of this paper and therefore, for the comparison with the shallow-water equations, we settle for patching the two pieces of the solution worked out in Sections 5 and 6.

The position of the head can be approximated using the integral formulation used in Section 6.1, while its structure is computed using the results of Section 6.2, where it has been shown that the head is wedge-shaped. The only unknown is the wedge angle $\chi$. We assume that the free boundary remains straight within the head $\left(x_{b} \leq x \leq x_{a}\right)$. By using the mass equation (44) and by approximating $M \approx \frac{1}{2}\left(x_{a}-x_{b}\right)^{2} \tan \chi$, we infer

$\tan \chi=\frac{2 \delta^{3}}{n \alpha^{2}} \frac{\xi_{a}^{2}}{\xi_{a}-\xi_{b}} t^{-(n-4) / 3}$,

showing that the wedge becomes increasingly acute with time. The angle $\chi$ is here only imposed by mass balance, whereas in finite-volume gravity currents investigated by McElwaine [46], the angle is controlled by dynamic conditions at the interface with the surrounding fluid.

\subsection{Comparison}

In order to compare the approximate similarity solution computed in Sections 5 and 6 with the similarity solution to the shallow-water equations, we need to compute the Boussinesq coefficient. After a few manipulations, integrating the velocity profile equation (37) leads to

$\gamma=\frac{9 \alpha^{2}-4}{8 \alpha^{2}}$

showing that the condition $\gamma \geq 1$ leads to $\alpha \geq 2$. Note that this condition provides a stronger constraint on $\alpha$ than Eq. (38). There is no immediate interpretation of these bounds, but recalling that the similarity form used for the velocity profile in Section 5.2 means that the velocity field must adapt instantaneously to any change in the flow depth, we can speculate that the flow must be rapid (i.e., supercritical) enough 

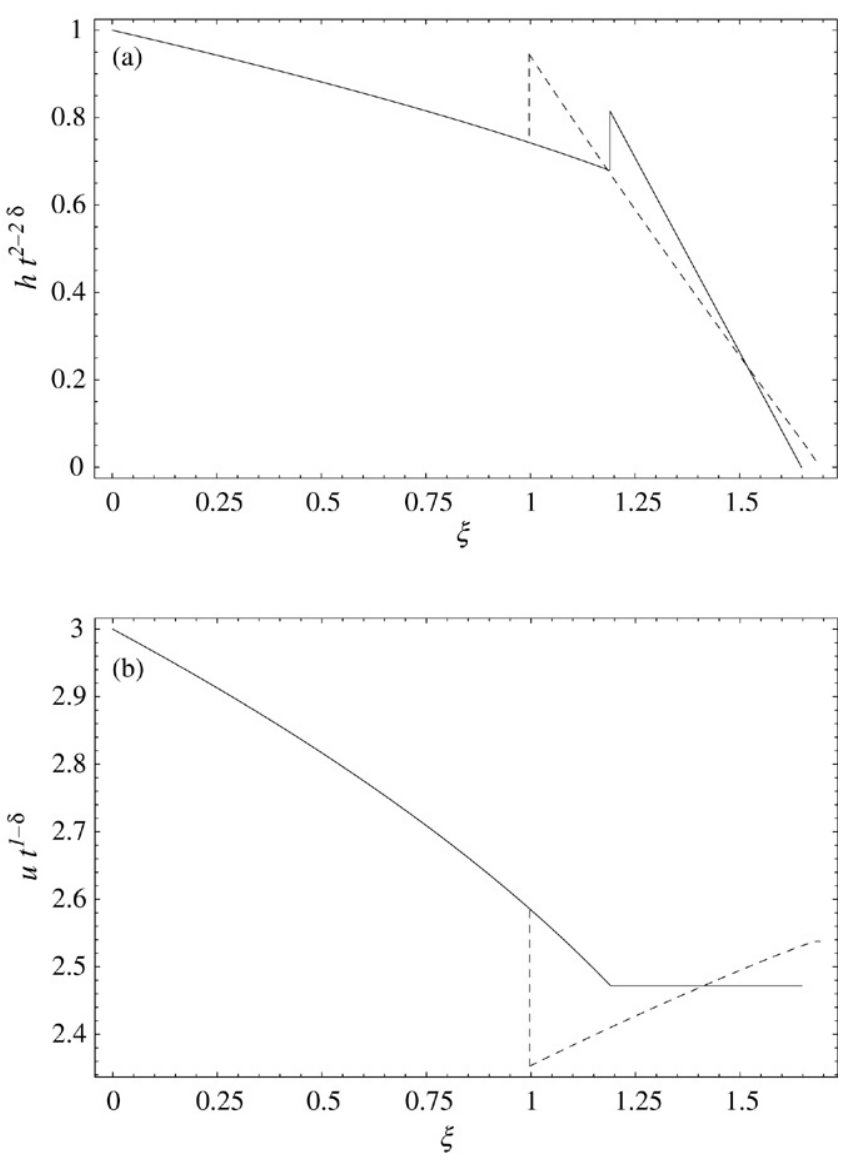

Fig. 16. (a) Flow depth profile, (b) velocity profile for the similarity solutions to the shallow-water equations (dashed line) and the approximate similarity solution Navier-Stokes equations in the high-Reynolds-number limit (solid line). Computations made for $\alpha=3, \beta=1, \delta=3 / 2(n=5 / 2), \gamma=77 / 72$.

for the information on flow depth changes to be promptly conveyed; indeed, in a shallow flow, information is transmitted on average at velocity $\sqrt{g h}(F r \pm 1)[66]$.

Fig. 16 shows the two solutions for the particular case $\alpha=3, \beta=1, \delta=3 / 2$, already addressed in Fig. 9, where the similarity solutions to the shallow-water equations were reported for two values of the Boussinesq coefficient: $\gamma=1$ and $\gamma=77 / 72$. As seen in Fig. 16, there is no difference between the similarity solutions to the shallow-water equations (4) and (5) and the shallow-wave equations (19) and (20) for the body, provided the Boussinesq coefficient is prescribed in advance. Differences arise for the head. Since there is no true similarity solution for the head velocity, we report the asymptotic value $\dot{x}_{f}$ computed using Whitham's approach in Section 6.1. The velocity profile computed using the shallowwater equations is not constant, but rises with increasing $\xi$; the departure from the asymptotic value, however, $\dot{x}_{f}$, remains small since it does not exceed $6 \%$.

\section{Concluding remarks}

In this paper, we have addressed the existence and features of similarity solutions to the shallow-water equations for describing high-speed (i.e., at high Reynolds numbers), nonBoussinesq flows along the horizontal plane. The velocity profile in the vertical direction may be nonuniform and in this case, the Boussinesq coefficient $\gamma=\overline{u^{2}} / \bar{u}^{2}$ exceeds unity.

The existence of similarity solutions has been proved for Boussinesq currents. In that case, the flow depth does not drop to zero, but to a finite value given by Eq. (2) $[25,26]$. From the mathematical point of view, it has also been demonstrated that the boundary value problem made up of the governing equations (4) and (5) and boundary conditions (9) and (10) is well-posed [51]. In Section 4, relying on a thorough analysis of the phase plane and the topological features of the governing equation, we have shown that the necessary condition for finding a similarity solution to the shallow-water equations for non-Boussinesq currents is that the front point $\mathrm{P}$ in the phase plane is a singular point. Indeed, no regular integral path, which represents a solution to the shallow-water equations, passes through P. However, when P is a singular point, there is an exceptional solution that passes through it. This exceptional solution is a piece of the solution sought. For $\mathrm{P}$ to be a singular point, the Boussinesq coefficient must drop to unity in the head. Another condition is placed on the growth rate of the current and the initial Froude number: for the similarity solution to be physically admissible, the current volume must grow as $t^{n}$, with $n \geq 1$, and the flow must be supercritical at the inlet $(F r>1)$. Except for currents with steady inflow $(n=1)$, the current head is separated from the body by a hydraulic jump and is wedgeshaped. The head is in a subcritical regime $(F r<1)$, while the body is in a supercritical regime $(F r>1)$. Since a jump is associated with a significant change in the velocity profile, it is also possible to construct solutions where the Boussinesq solution is unity within the leading edge, but is in excess of unity within the body [4].

Strikingly, these severe restrictions on the existence of similarity solutions in the non-Boussinesq case differ substantially from the Boussinesq case, where Gratton and Vigo [25] showed that solutions of this kind exist for a wide range of flow conditions, including subcritical entrance conditions and low growth rates $n<1$. As pointed out by Gratton and Vigo [25], the non-Boussinesq case could be considered as a limiting Boussinesq regime when the Froude number at the front tends to infinity, and in this perspective, one would expect to retrieve nearly the same solutions. This is not the case. As for tension-driven coating films, we find that imposing zero or finite values on the flow depth at the front can lead to significantly different results.

To make this difference between Boussinesq and nonBoussinesq currents clear, we have refined our analysis by seeking similarity solutions to the Navier-Stokes equations in the high-Reynolds-limit number (Euler equations). A particular difficulty stems from the shallowness approximation used in the derivation of the shallow-water equations. Since this approximation is not valid within the leading edge, we distinguished the head and body dynamics:

- Similarity solutions were derived for the body and were fully consistent with those worked out for the shallow-water equations (27) and (28). This consistency results directly from our primary choice of the similarity forms (27) and (28) 
used for solving the shallow-wave equations (19) and (20). Indeed, in these similarity forms, we did impose a constant Froude number. Physically, this result is in agreement with experimental [45] and intuitive observations since from a force balance in the momentum equation, we can interpret the similarity phase as a flow regime characterized by a balance between inertia and the pressure gradient

$$
u \frac{\partial u}{\partial x} \sim g \frac{\partial h}{\partial x},
$$

which leads to finding a nearly constant Froude number. Similarity solutions to Eqs. (19) and (20) exist provided that the Froude number is in excess of $4 \sqrt{2} / 3 \approx 1.88$. As for the shallow-water equations, we retrieve the condition that flows must be supercritical. There is, however, a significant difference between the limiting Froude number for a similarity solution to exist: $F r \geq 1$ for the shallow-water equations versus $4 \sqrt{2} / 3$ for the shallow-wave equations. One question which was not addressed concerns the flow stability. Stability analysis of the shallow-water equations shows that steady uniform flows become unstable for $\mathrm{Fr}>$ 2 [67], but since this analysis cannot easily be extrapolated to transient flows, we do not investigate this question further.

- We failed to find a similarity solution representing the head behavior, but we found approximate similarity solutions when the volume growth rate $n$ satisfies $n<4$. In this case, the front acceleration tends to zero when time elapses so that we can expand the velocity into a time series and retain the terms at the leading order. Analyzing the governing equations showed that the flow must be rotational and that the head is wedge-shaped. The shape is in fairly good agreement with that computed using the shallow-water equations. The leading edge is associated with a pair of vortices, one located at the leading edge and another one at the rear of the head. This wedge structure, including vortices, has been observed in experiments on gravity currents in tanks. In experiments conducted by Simpson $[58,59]$, the development of the flow patterns was made visible using a blend of dense fluid and fine aluminum particles: a stretching vortex occupying the tip region was clearly observed at the leading edge and produced an intense roll-up of fine aluminum particles, which makes it possible to visualize the streamlines and the two vortices; in the upper part of the head, a counter-clockwise rotating vortex occurred. Our own experiments on finite-volume gravity currents moving down a slope also revealed that the particle cloud was composed of two evident eddies [2]: when the surge involving a glass-bead suspension in water moved from left to right, we observed a small vortex ahead of the front, spinning clockwise, and a large counter-clockwise eddy occupying most of the surge volume. In his seminal paper, Benjamin [7] supplemented the earlier heuristical analysis of von Kármán, demonstrating that a steady front makes an angle of $\pi / 3$ with respect to the horizontal: Benjamin provided an approximate analytical solution describing the shape for the lock-exchange problem when the flow depth is half the total depth and again found that the front angle was $\pi / 3$.
Recently, McElwaine [46] has extended Benjamin's results by considering steady finite-volume currents down a steep slope, which experience resistance from the surrounding fluid. $\mathrm{He}$ also found that the front makes an angle $\pi / 3$ with the bottom line. Our result contrasts with the earlier findings: the front angle is not constant, but varies with time. Therefore, there appear to be significant changes in dynamics in the front angle between steady and timedependent flow conditions. This observation may have a potential impact since until now, most models have used a constant-Froude-number boundary condition even though the flow is not steady.

When hydraulic resistance becomes significant or when the surrounding fluid exerts a sufficient resistance to the surge spreading, the shape of the front bulges out. A typical example of the rising influence of hydraulic resistance and its consequence on the front shape is provided in the paper by [30], where the motion of the surge resulting from the collapse of a fluid volume (dam break) is investigated: when the surge starts being affected by drag, it becomes blunt-nosed with $H(\xi) \propto\left(\xi_{f}-\xi\right)^{1 / 2}$, rather than adopting a linear profile close to the front; indeed, the current develops an increased streamwise pressure gradient to counter the drag force exerted by the bottom. In this respect, we should expect that at late times, drag also arises in the particular context investigated here since the flow becomes increasingly thin: the front angle $\tan \chi$ varies as $t^{-2}$ according to (52).

\section{Acknowledgements}

The work presented here was supported by the Swiss National Science Foundation under grant number 200021105193/1, the competence center in Mobile Information and Communication Systems (supported by the Swiss National Science Foundation under grant number 5005-67322), and specific funds provided by EPFL (vice-présidence à la recherche).

\section{Appendix A. Similarity solutions}

\section{A.1. Similarity and resulting governing equation}

The shallow-water equations admit many symmetry groups [15,36], including the stretching group that enables us to derive similarity solutions. A particularly appropriate form for working out similarity solutions is the following:

$\bar{u}=\delta \xi t^{\delta-1} V(\xi)$,

$h=\delta^{2} \xi^{2} t^{2(\delta-1)} Z(\xi)$,

where we have introduced the similarity variable

$\xi=\frac{x}{t^{\delta}}$,

with $\delta$ a positive constant. There are many possible ways of constructing similarity solutions. In the class of problems dealt with here, symmetry analysis usually suggests seeking similarity relations in the form $\bar{u}=t^{c_{1}} \hat{V}(\xi)$ and $h=t^{c_{2}} \hat{Z}(\xi)$, 
where $c_{1}$ and $c_{2}$ are two constants $[9,19,22]$. It is sometimes more advantageous to write down these relations in the form $\bar{u}=t^{c_{1}} \xi^{c_{3}} \tilde{V}(\xi)$ and $h=t^{c_{2}} \xi^{c_{4}} \tilde{Z}(\xi)$, where $c_{3}$ and $c_{4}$ are two other constants, $\tilde{V}(\xi)=\xi^{-c_{3}} \hat{V}(\xi)$ and $\tilde{Z}(\xi)=\xi^{-c_{4}} \hat{Z}(\xi)$, so that the governing equations (4) and (5) can be transformed into an autonomous differential equation [i.e., in Eq. (15), $\xi$ does not appear], which makes it then possible to use the phase-portrait techniques. In doing so, we find $c_{1}=\delta-1, c_{2}=2 \delta-2$, $c_{3}=1, c_{4}=2$. Note that it is also advantageous to do the same with parameter $\delta$ and include it in the similarity relations so that we can get rid of it in the final governing equation (15), which simplifies a great deal the examination of the critical points of the phase plane.

Substituting the similarity forms into the governing equations (4) and (5), we obtain two ordinary first-order differential equations for $Z$ and $V$ that can be cast in a matrix form

$\mathbf{M}(V, Z) \frac{\mathrm{d} \mathbf{w}}{\mathrm{d} \xi}+\frac{Z}{\delta \xi} \mathbf{S}(V, Z)=0$,

with $\mathbf{w}=[Z, V]^{\mathrm{T}}$,

$\mathbf{M}=\left[\begin{array}{cc}V-1 & Z \\ (\gamma-1) V^{2}+Z & Z(V(2 \gamma-1)-1)\end{array}\right], \quad$ and

$\mathbf{S}=\left[\begin{array}{c}3 V \delta-2 \\ 2 \delta Z+V(V(4 \gamma-3) \delta-1)\end{array}\right]$.

The determinant of the matrix $\mathbf{M}$ is $\operatorname{det} \mathbf{M}=\delta Z(Z-I(V))$, with

$I(V)=1+(V-2) V \gamma$.

In the phase plane, we introduce the curve $C_{I}$ of equation $Z=$ $I(V)$. This curve plays a very important role since usually no continuous integral path can reach or cross it. Very occasionally, when the cofactor matrix is also zero (see below), crossing or reaching $C_{I}$ is permitted. In the usual case, when no continuous path can cross $C_{I}$, one can sometimes use the Rankine shock conditions (11) and (12) to work out discontinuous solutions (see Appendix A.2). Note that along the $V$-axis $(Z=0)$, the determinant is also zero, but its role is less significant since this line lies on the borderline of the first quadrant $(V \geq 0$, $Z \geq 0$ ) and only the integral paths in this domain are physically interesting.

In the regions where $\operatorname{det} \mathbf{M}$ is nonzero, the system of Eq. (A.1) can be inverted to provide

$$
\begin{aligned}
& \xi \frac{\mathrm{d} Z(\xi)}{\mathrm{d} \xi}=\frac{F(V, Z)}{\delta(Z-I(V))}, \\
& \xi \frac{\mathrm{d} V(\xi)}{\mathrm{d} \xi}=\frac{G(V, Z)}{\delta(Z-I(V))},
\end{aligned}
$$

with

$$
\begin{aligned}
F(V, Z)= & -Z(2 Z \delta+V(-2 V \delta \gamma+4 \gamma+3 \delta-3)-2), \\
G(V, Z)= & Z(2-(V+2) \delta) \\
& +V(V(2 \gamma+((V-4) \gamma+3) \delta-3)+1) .
\end{aligned}
$$

Instead of solving this system of differential equations, we form the ratio of the two equations to arrive at a single ordinary differential equation

$\frac{\mathrm{d} Z}{\mathrm{~d} V}=\frac{F(V, Z)}{G(V, Z)}$.

This allows us to work in the $V-Z$ plane, as discussed at length in Section 4.

When det $\mathbf{M}$ is zero, the system may have solutions if the determinant of the cofactor matrix

$\mathbf{N}=\left[\begin{array}{cc}V-1 & 3 V \delta-2 \\ (\gamma-1) V^{2}+Z & 2 \delta Z+V(V(4 \gamma-3) \delta-1)\end{array}\right]$,

is also zero. In the space $(V, Z)$, the locus of the points for which $\operatorname{det} \mathbf{N}=0$ is a continuous curve $C_{G}$ of the equation

$Z=J(V)=\frac{V\left(\gamma \delta V^{2}+(-4 \delta \gamma+2 \gamma+3 \delta-3) V+1\right)}{(V+2) \delta-2}$.

Note that the curve $C_{G}$ is also the locus of points where $G(V, Z)=0$, which means that:

- Any integral path crossing $C_{G}$ admits a vertical tangent at the point of crossing.

- The points where $C_{G}$ and $C_{I}$ coincide are singular solutions to Eq. (A.1).

To close our boundary value problem, we need to specify the boundary conditions. The boundary conditions (9) and (10) at the front impose

$Z\left(\xi_{f}\right)=0$ and $V\left(\xi_{f}\right)=1$,

where $\xi_{f}$ denotes the front position. At the source, Eqs. (6)-(8) imply that $Z$ and $V$ tend toward infinity as follows:

$Z \propto \frac{\beta}{\delta^{2} \xi^{2}} \quad$ and $\quad V \propto \frac{\alpha \sqrt{\beta}}{\delta \xi} \quad$ when $\xi \rightarrow 0$.

Since the solution may admit discontinuities, we supplement the following condition derived from Eq. (6), which ensures that the mass balance is not violated:

$\int_{0}^{\xi_{f}} \xi^{2} Z(\xi) \mathrm{d} \xi=\delta^{-2} A$

When there is no discontinuity, this equation is redundant with Eq. (A.5).

Eventually, note that the coefficient $\delta$ is fixed by the volume growth rate: replacing $h$ and $u$ by their similarity forms into the initial conditions $(6)$, we find $\delta=(n+2) / 3$.

\section{A.2. Flow discontinuity in the phase plane}

The two Eqs. (11) and (12) involve three unknown variables and therefore are not closed. To close the equations, we use the volume balance equation (A.6). When a shock occurs and if we know the flow variables $Z_{1}$ and $V_{1}$ upstream (respectively, downstream) of the shock, we can solve the shock equations (11) and (12) to determine the shock velocity $\sigma$ and a curve referred to as the shock curve $V_{2}\left(Z_{2} \mid Z_{1}, V_{1}\right)$, which is the locus of all the points satisfying the jump conditions (11) and (12). Solving this system of equations, we derive the shock velocity 
and the variation in upstream velocity $V_{2}$ with upstream flow depth $Z_{2}$ :

$$
\begin{aligned}
& \sigma=\frac{\gamma}{\beta} V_{1} \pm \frac{1}{\beta} \sqrt{\frac{1}{2}\left(\beta\left(Z_{1}+Z_{2}\right)+2(\gamma-1) V_{1}^{2}\right) \frac{Z_{2}}{Z_{1}}}, \\
& V_{2}\left(Z_{2} \mid Z_{1}, V_{1}\right)= \frac{V_{1}}{\beta} \pm \frac{\left(Z_{2}-Z_{1}\right)}{\beta} \\
& \times \sqrt{\frac{1}{2} \frac{\beta\left(Z_{1}+Z_{2}\right)+2(\gamma-1) V_{1}^{2}}{Z_{1} Z_{2}}},
\end{aligned}
$$

with $\beta=\gamma+(1-\gamma) \frac{Z_{2}}{Z_{1}}$.

In the derivation of (A.7) and (A.8), we have assumed that the Boussinesq coefficient is the same either side of the jump. Similar albeit far more complicated relations can be derived when the jump is associated with a modification in the velocity profile; we will not report these relations here since they do not entail any change when we compute the shock from upstream to downstream (although the converse is not true).

Since there is a quadratic dependence on velocity in (11) and (12), we actually find two shock curves, but a single one is physically admissible by requiring that energy dissipation through the shock be positive.

\section{Appendix B. Critical points and separatrix}

To understand the phase portraits, it is worth recalling some basic properties of the singular points. Through a regular (i.e., nonsingular) point, a single integral path can pass and all the curves in its close neighborhood have the same behavior. In contrast, a singular point can be crossed either by an infinity of curves (if the point is a node) or a single curve (if it is saddle); here, we will not mention the case of focus points. The singularity type can usually be obtained by linearizing Eqs. (A.2) and (A.3) around a singular point $\left(V_{0}, Z_{0}\right)[6,38]$ :

$\frac{\mathrm{d}}{\mathrm{d} \xi}\left[\begin{array}{c}V-V_{0} \\ Z-Z_{0}\end{array}\right]=\left[\begin{array}{ll}\partial_{V} F & \partial_{Z} F \\ \partial_{V} G & \partial_{Z} G\end{array}\right] \cdot\left[\begin{array}{c}V-V_{0} \\ Z-Z_{0}\end{array}\right]$.

Note that since the singular behavior results from $F$ and $G$ vanishing at $\left(V_{0}, Z_{0}\right)$, it is no use considering the denominator $Z(Z-I(v))$ in the linearization. We are then seeking solutions in the form $\mathbf{v}=\mathbf{v}_{0} \mathrm{e}^{-\lambda t}$. It is straightforward to deduce that $\lambda$ must be an eigenvalue of the linear system above, while $\mathbf{v}_{0}$ is an eigenvector.

When the two eigenvalues are real and of the same sign, the singular point is a node, and when $\xi \rightarrow \infty$ or $\xi \rightarrow-\infty$, the two solutions converge to the singular point by following the directions given by the eigenvectors (see Fig. B.1). These solutions are special solutions called separatrices since they can usually be used to delineate different regions in the phase plane. If we now consider a point in the immediate vicinity of the singular point and integrate Eqs. (A.2) and (A.3) from this point, the resulting integral path will pass through the singular point, and its tangent at that point is collinear with one of the eigenvectors. This means that one of the special curves is also the limiting curve at which all the integral paths passing
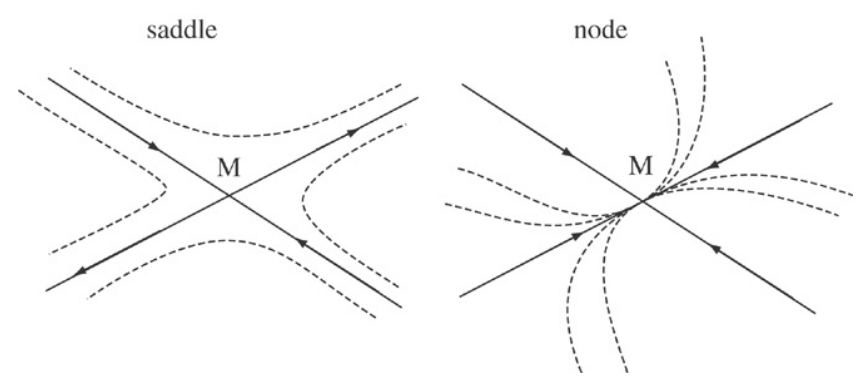

Fig. B.1. Saddle and nodes in the phase plane. The solid lines represent the special solutions; the arrows indicate the direction pointed by increasing $\xi$. The dashed lines represent the integral paths.

through the singular point are tangent (except for the other special solution).

When the two eigenvalues are real and of opposite sign, the singular point is a saddle, and when $\xi \rightarrow \infty$ or $\xi \rightarrow-\infty$, one of the two solutions converges to the singular point by following the direction given by one of the eigenvectors, while the other solution diverges (see Fig. B.1). The solutions are also special solutions and their curves are called separatrices. For a saddle, there is only one curve passing through it (one of the separatrices); all integral paths are deflected when approaching this point.

For both saddle and node points, separatrices play a key role. Their equations can be derived theoretically by seeking the symmetry groups leaving Eq. (15) invariant [8,19] or numerically by using L'Hôpital's rule [19]. Basically, the latter technique involves expanding the different terms in Eq. (15) into a power series of $F$. Let us refer to the equation of the separatrix by $Z=Z_{s}(V)$. Since $Z_{s}(V)$ is an exceptional solution of Eq. (15), we can write

$$
\begin{aligned}
Z_{s}^{\prime}\left(V_{0}\right)\left(V-V_{0}\right)+\frac{1}{2} Z_{s}^{\prime \prime}\left(V_{0}\right)\left(V-V_{0}\right)^{2}+\cdots \\
=\frac{\left(V-V_{0}\right) \mathrm{D}^{(1)} F+\frac{1}{2}\left(V-V_{0}\right)^{2} \mathrm{D}^{(2)} F+\cdots}{\left(V-V_{0}\right) \mathrm{D}^{(1)} G+\frac{1}{2}\left(V-V_{0}\right)^{2} \mathrm{D}^{(2)} G+\cdots}
\end{aligned}
$$

with $\mathrm{D}^{(1)}=\partial_{V}+Z_{s}^{\prime} \partial_{Z}, \mathrm{D}^{(2)}=\partial_{V V}+2 Z_{s}^{\prime} \partial_{V Z}+Z_{s}^{\prime 2} \partial_{Z Z}+$ $Z_{s}^{\prime \prime} \partial_{Z}$, etc. the total derivatives of order 1,2 , etc. Then collecting the coefficients associated with the same power of $V-V_{0}$ makes it possible to find a Taylor series of the separatrix. Note that there are two possible values for the first-order term and these values correspond to the slope of the eigenvectors of the matrix of Eq. (B.1). We end up with a power series in the form

$$
Z_{s}(V)=Z_{0}+Z_{s}^{(1)}\left(V-V_{0}\right)+\frac{1}{2} Z_{s}^{(2)}\left(V-V_{0}\right)^{2}+\cdots,
$$

with

$$
\begin{aligned}
& Z_{s}^{(1)}=Z_{s}^{\prime}\left(V_{0}, Z_{0}\right) \\
& =\frac{\partial_{Z} F-\partial_{V} G \pm \sqrt{\left(\partial_{Z} F\right)^{2}-2 \partial_{V} G \partial_{Z} F+\left(\partial_{V} G\right)^{2}+4 \partial_{V} F \partial_{Z} G}}{2 \partial_{Z} G}, \\
& Z_{s}^{(2)}=Z_{s}^{\prime \prime}\left(V_{0}, Z_{0}\right) \\
& =\frac{-\left(Z_{s}^{(1)}\right)^{3} \partial_{Z Z} G+\left(Z_{s}^{(1)}\right)^{2}\left(\partial_{Z Z} F-2 \partial_{V Z} G\right)+2 Z_{s}^{(1)}\left(\partial_{V Z} F-\partial_{V V}\right)+\partial_{V V} F}{-\partial_{Z} F+2 \partial_{V} G+3 Z_{s}^{(1)} \partial_{Z} G} .
\end{aligned}
$$


When several singular points lie in the same region, they share the same separatrices. For instance, in Fig. 5, $S_{\mathrm{AB}}$ is a separatrix for the singular points $\mathrm{A}_{\gamma}$ and $\mathrm{B}_{\gamma}$. It is worth noting that the numerical computation of the Taylor series decomposition of $Z_{s}$ may encounter severe difficulties when two neighboring points are not of the same topological nature (which is the usual case). A priori, there is no specific reason for the separatrix equation to be decomposable into a Taylor series. Fig. B. 2 shows how accurate a truncated Taylor series decomposition is when its order is increased. We first computed the Taylor series of separatrix $S_{\mathrm{AB}}$ for $\gamma=1$ and $\delta>1$ by starting from point $A_{1}(2,1)$, which is a node. The separatrix passes through point $\mathrm{B}_{1}(1,0)$, which is a saddle; the values of $Z_{s}$ at $V=1$ computed using Eq. (B.2) can then be compared to the expected value 0 and give an idea of the accuracy of this expansion. Fig. B.2(a) shows the value $Z(1)$ for order 26. It is seen that for $\delta<5 / 4$, the accuracy increases with increasing order, as expected. However, for $\delta>5 / 4$, the converse can be observed, which shows that the series diverges. The same exercise was repeated by computing the separatrix equation starting from point $\mathrm{B}_{1}$ and evaluating the expansion accuracy at point $A_{1}$. Fig. B.2(b) shows that for $\delta>5 / 4$, accuracy is enhanced by increasing the series order, while the Taylor series diverges for $\delta<5 / 4$.

\section{Appendix C. Construction of the similarity solution in the phase plane}

\section{C.1. Usual computational procedure}

The solution in the phase plane can be found by solving Eq. (15) numerically. In practice, this was achieved by proceeding as follows [4]:

(1) We selected a pair of points $\left(V_{S}, Z_{S}\right)$ standing for the source point and such that $Z_{S}=\alpha^{-2} V_{S}^{2}$ so that the boundary condition (A.6) is satisfied. Usually, taking $V_{S}$ on the order of $10^{4}$ ensured accuracy to within $10^{-3}$.

(2) The ordinary differential equation (15) was then solved using standard techniques (i.e., the predictor-corrector Adams method). The resulting solution was plotted in the phase plane as an integral path $\mathcal{C}$ of equation $Z=Z(V)$.

(3) On some occasions, the integral path crossed or came close to the critical curve $C_{I}$. In that case, the numerical solution started to diverge: a discontinuous solution (strong shock) was then envisaged. The integral path was made up of a continuous path on either side of $C_{I}$, while the endpoints of these pieces were linked together by the shock conditions (A.7) and (A.8). See Appendix C.2.

(4) To resolve the dependence on $\xi$ and determine $V(\xi)$ and $H(\xi)$, we first integrated Eq. (A.3) along the integral path $\mathcal{C}$ from $S$ to any point $M$ (with some restrictions on $M$ if the solution crossed the critical curve $C_{I}$ ) and we obtained an equation in the form

$$
\xi_{M}=\xi_{S} \exp \left(-\int_{M}^{S} \frac{I(V, Z(V))}{G(V, Z(V))} \mathrm{d} V\right)
$$
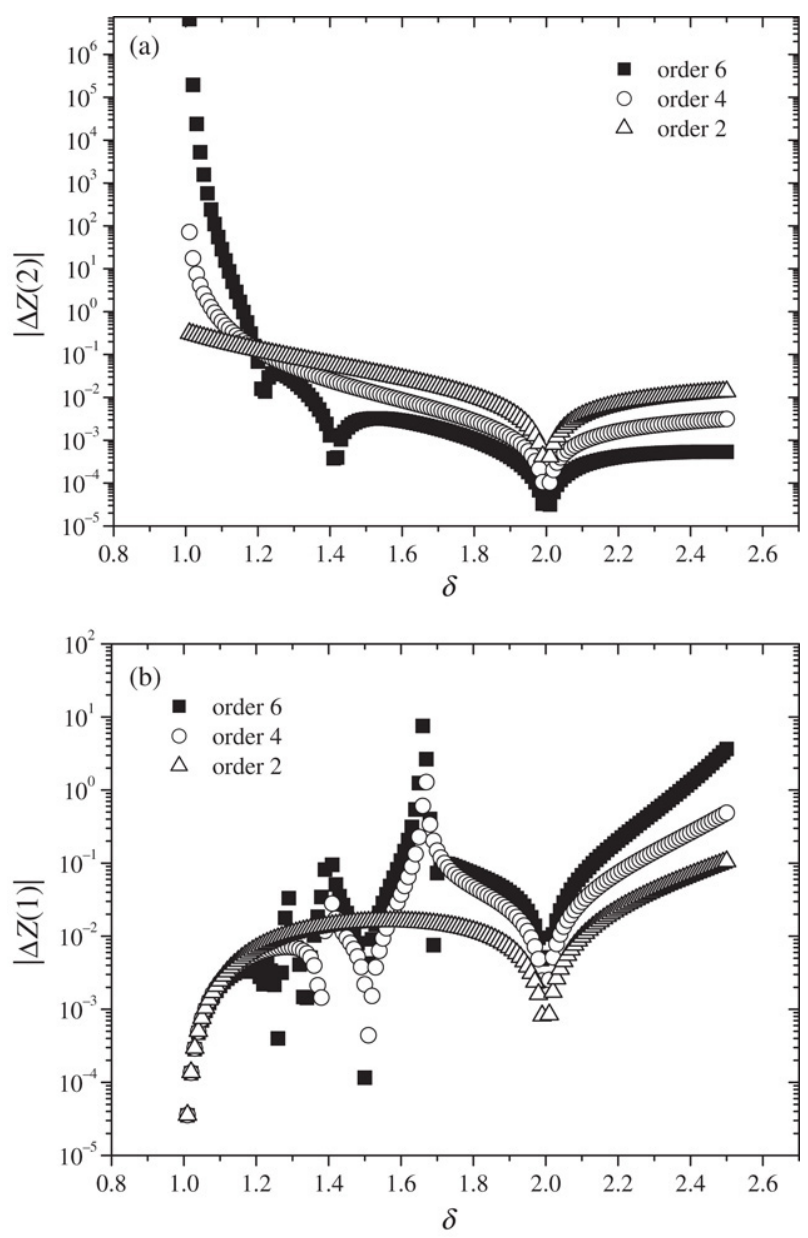

Fig. B.2. Variation in the $Z(1)$ value computed using the Taylor expansion given by L'Hôpital's rule to different orders when the $\delta=(2+n) / 3$ value is varied.

which allowed us to compute the value $\xi_{M}$ associated with any point $M\left(V_{M}, Z_{M}=Z\left(V_{M}\right)\right)$. Taking into account the asymptotic behavior when $\xi \rightarrow 0$ given by Eq. (A.5), we were able to get rid of the terms representing the behavior close to the source in order to obtain a closed form for the coordinates of M. Note that when $\alpha=2(9-8 \gamma)^{-\frac{1}{2}}$, this computation can be done analytically:

$\xi_{M}=\left(\frac{2 V_{M} \delta-2}{3 \delta}\right)^{\delta-1} \frac{\alpha \sqrt{\beta}}{\delta V_{M}^{\delta}}$.

\section{C.2. Numerical treatment of the discontinuities}

We refer to point $\mathrm{E}\left(V_{E}, Z_{E}\right)$ as the point at which the shock occurs. First of all, note that:

- It is not possible to directly relate $\mathrm{P}$ and $\mathrm{E}$ using the shock conditions (A.7) and (A.8) because a hydraulic jump cannot form between a dry bed and the current.

- Since no regular solution except the trivial solution $Z=$ 0 passes through $\mathrm{P}$, there is no other way of joining the subcritical and supercritical branches of $\mathcal{C}$ since the subcritical branch does not exist. 
The path $\mathrm{E}^{\prime} \rightarrow \mathrm{P}$ along the separatrix $S_{\mathrm{AB}}$ must be integrated numerically.

To locate the position of $\mathrm{E}$ along the integral path, we used a trial-and-error procedure. On the integral path coming from the source point, we first guessed the position of point $\mathrm{E}$ in the phase plane $V-Z$. We deduced the position of $\mathrm{E}^{\prime}$ by seeking the intersection point between the proper shock curve and the separatrix $S_{\mathrm{AB}}$, then we computed the relations $V(\xi)$ by solving Eq. (A.3) numerically on either piece of the solution. The volume of the current was then computed by integrating $\int_{0}^{\xi_{f}} \xi^{2} Z(\xi) \mathrm{d} \xi$ and compared with the expected value $A \delta^{-2}$ [see Eq. (A.6)]. The position of $E$ was then varied until the computed and expected total volumes coincided.

\section{Appendix D. Ritter solutions}

For $\delta=n=1$ and $\gamma=1$, the critical curves $C_{I}$, and $C_{F}$, and $C_{G}$ form a single curve of equation $Z=(V-1)^{2}$; the functions $F$ and $G$ simplify a great deal:

$F=2 Z\left((V-1)^{2}-Z\right)$ and $G=V\left((V-1)^{2}-Z\right)$,

so that Eq. (15) is transformed into a simple equation

$\frac{\mathrm{d} Z}{\mathrm{~d} V}=\frac{2 Z}{V}$.

This shows that the equations of the regular integral paths are of the form $Z=k_{1} V^{2}$, with $k_{1}$ an integration factor. The boundary conditions at the source yield $k_{1}=\alpha^{-2}$. Using Eqs. (A.2) and (A.3) to resolve the dependence on $\xi$, we find

$\xi \mathrm{d} Z+2 Z \mathrm{~d} \xi=0$

which provides

$Z=\beta \xi^{-2}, \quad$ hence $V=\sqrt{\beta} \alpha \xi^{-1}$,

when the boundary conditions (9) are taken into account. In the phase plane, the corresponding curves are parabolas $\mathcal{P}$ of equation $Z=(V / \alpha)^{2}$.

As is seen in Fig. 8, curves of this type do not satisfy the boundary conditions at the front. However, they meet the critical curve $C_{I}$ at point

- $\mathrm{E}^{\prime}$ of coordinates $\left(\alpha /(\alpha+1),(1+\alpha)^{-2}\right)$ associated with $\xi_{e}=\sqrt{\beta}(1+\alpha)$ when $\alpha<1$.

- E of coordinates $\left(\alpha /(\alpha-1),(1-\alpha)^{-2}\right)$ associated with $\xi_{e}=\sqrt{\beta}(1-\alpha)$ when $\alpha>1$.

This coalesced $C_{I}$ curve is a singular solution to Eq. (A.1), which corresponds to $\alpha=1$. Indeed, along this curve, we have $\operatorname{det} \mathbf{N}=0$ and $\operatorname{det} \mathbf{M}=0$. This implies that $Z=(V-1)^{2}$ and Eq. (A.1) reduces to

$3 \xi \mathrm{d} V+(3 V-2) \mathrm{d} \xi=0$,

which, when integrated, provides

$V=\frac{2}{3}+\frac{k_{2}}{\xi}$ where $k_{2}$ is an integration constant. When $\alpha>1$, using the coordinates of $\mathrm{E}$, we deduce the value of $k_{2}$ :

$k_{2}=\frac{2}{3} \sqrt{\beta} \alpha$.

Returning to the dimensionless physical variables, we find

$\bar{u}=\xi V=\frac{2}{3}(\xi+\alpha \sqrt{\beta})$,

$h=\xi^{2} Z=\xi^{2}(V-1)^{2}=\frac{1}{9}(\xi-2 \alpha \sqrt{\beta})^{2}$

for $\xi \geq \xi_{e}$, while for $\xi<\xi_{e}$, we have

$\bar{u}=\alpha \sqrt{\beta}$,

$h=\beta$.

When $\alpha \rightarrow 1$, we retrieve the usual Ritter solution found by Ritter [52] in his analysis of the dam-break problem. Note that this problem, where an infinite volume of fluid retained by a dam is unleashed at time $t=0$, is nearly equivalent to a constant-discharge problem because if we examine what occurs at $x=0$ (hence $\xi=0$ ) for the Ritter solution, we find that the flow depth and velocity are constant, and hence the flow rate is in turn constant. The equivalence is, however, not complete since the flow geometries differ slightly.

When $\alpha<1$, we can also construct a mathematical solution, but this solution is nonphysical. Indeed, in that case, the integration constant $k_{2}$ is $(2-\alpha) \sqrt{\beta} / 3$, which means that the velocity

$\left|V-\frac{2}{3}\right|=\frac{(\alpha-2) \sqrt{\beta}}{3 \xi}$,

tends to $2 / 3$ and the front point $\mathrm{P}(V=1)$ is never reached.

\section{Appendix E. Scaling of the Navier-Stokes equation}

The governing equations are given by the Navier-Stokes equations. These equations can be simplified a great deal by focusing on shallow inertial flows, i.e., keeping only the terms whose order of magnitude is $O\left(\epsilon^{0}\right)$.

The characteristic streamwise and vertical velocities, the timescale, and the typical pressure are referred to as $U_{*}, V_{*}, T_{*}$, and $P_{*}$, respectively. Moreover, in addition to the length scale ratio $\epsilon$, we introduce the following dimensionless numbers that characterize free-surface, gravity-driven flows: the flow Reynolds number $R e=\rho U_{*} H_{*} / \mu$ and the Froude number $F r=U_{*} / \sqrt{g H_{*}}$. Finally, the following dimensionless variables will be used: $u=\hat{u} / U_{*}, v=\hat{v} / V_{*}, x=\hat{x} / L_{*}, y=\hat{y} / H_{*}$, $t=\hat{t} / T_{*}$, and $p=\hat{p} / P_{*}$, where the hat refers to dimensional variables. Natural choices for $T_{*}$ and $P_{*}$ are $T_{*}=L_{*} / U_{*}$ and $P_{*}=\rho g H_{*}$ since we expect that, to leading order, the pressure adopts a hydrostatic distribution. If we define the vertical velocity scale as $V_{*}=\epsilon U_{*}$, the mass balance equation takes the following dimensionless form:

$\frac{\partial u}{\partial x}+\frac{\partial v}{\partial y}=0$ 
At high speeds, there is a balance between inertia and the pressure gradient, which leads us to define the velocity scale as: $U_{*}=\sqrt{g H_{*}}$. In this case, the order of magnitude of the viscous stress is $\rho g H_{*} O\left(\epsilon^{-1} R e^{-1}\right)$. For the viscous contribution to the momentum balance to be neglected compared to inertial and pressure terms, the following conditions must be satisfied: $\epsilon \operatorname{Re} \gg 1$. In this case, we also have: $F r=O(1)$. We refer to this regime as the inertial flow regime. The governing equations take the following dimensionless form:

$$
\begin{aligned}
\frac{\partial u}{\partial t}+u \frac{\partial u}{\partial x}+v \frac{\partial u}{\partial y}=-\frac{\partial p}{\partial x} & +\lambda\left(\epsilon^{2} \frac{\partial^{2} u}{\partial x^{2}}+\frac{\partial^{2} u}{\partial y^{2}}\right), \\
\epsilon^{2}\left(\frac{\partial v}{\partial t}+u \frac{\partial v}{\partial x}+v \frac{\partial v}{\partial y}\right)= & -1-\frac{\partial p}{\partial y} \\
& +\lambda \epsilon\left(\epsilon^{2} \frac{\partial^{2} v}{\partial x^{2}}+\frac{\partial^{2} v}{\partial y^{2}}\right),
\end{aligned}
$$

where $\lambda=(\epsilon R e)^{-1} \ll 1$.

\section{E.1. Governing equations for the body}

When $\lambda \ll 1$ and $\epsilon \ll 1$, then we can get rid of higher-order terms in Eqs. (E.2) and (E.3) and we end up with the so-called shallow-wave equations [66]

$$
\begin{aligned}
& \frac{\partial u}{\partial t}+u \frac{\partial u}{\partial x}+v \frac{\partial u}{\partial y}=-\frac{\partial p}{\partial x}, \\
& 0=-1-\frac{\partial p}{\partial y} .
\end{aligned}
$$

\section{E.2. Governing equations for the head}

When $\lambda \ll 1$, but $\epsilon=O(1)$, we retrieve the dimensionless Euler equations

$$
\begin{aligned}
& \frac{\partial u}{\partial t}+u \frac{\partial u}{\partial x}+v \frac{\partial u}{\partial y}=-\frac{\partial p}{\partial x}, \\
& \frac{\partial v}{\partial t}+u \frac{\partial v}{\partial x}+v \frac{\partial v}{\partial y}=-1-\frac{\partial p}{\partial y} .
\end{aligned}
$$

\section{Appendix F. Irrotational solution}

Our problem boils down to finding the stream function for an inviscid irrotational flow in a wedge of angle $\chi$. We shall see that the only solution to this problem is the trivial solution $\psi_{0}=0$ within the tip region. Indeed, the complex potential $w(z)=\phi_{0}+\mathrm{i} \psi_{0}$, with $z=x+\mathrm{i} y$ and $\phi_{0}$ the potential function, is analytical for an irrotational inviscid flow. If we focus our attention on the close neighborhood of the leading edge, the free surface at order $O\left(t^{0}\right)$ appears as a stationary line, making an angle $\chi$ with respect to the bed. We solve the equation $\Delta \psi_{0}=0$ within a wedge. The contour line of this wedge is the streamline $\psi_{0}=0$. Using the conformal transformation $Z=X+\mathrm{i} Y=\mathrm{e}^{z}$, the wedge is transformed into an infinite strip; the Laplace equation reads $\psi_{0, Y Y}=0$ in the $Z$-plane and the boundary conditions are the following: $\psi_{0}=0$ on $Y=0$ and $Y=\chi$.
Hence, we obtain $\psi_{0}=0$. This result might be anticipated with physical intuition because the only possibility for observing an irrotational flow in a wedge is assuming that there is a sink at the edge. The only way to overcome this paradoxical result is to assume that there are sources of vorticity within the tip region.

\section{References}

[1] M. Abramowitz, I.A. Stegun, Handbook of Mathematical Functions, National Bureau of Standards, Washington, 1964.

[2] C. Ancey, Powder-snow avalanches: Approximation as non-Boussinesq clouds with a Richardson-number-dependent entrainment function, J. Geophys. Res. 109 (2004) F01005.

[3] C. Ancey, Plasticity and geophysical flows: A review, J. Non-Newtonian Fluid Mech. (2006), in press (doi:10.1016/j.jnnfm.2006.05.005).

[4] C. Ancey, S. Cochard, S. Wiederseiner, M. Rentschler, Front dynamics of supercritical non-Boussinesq gravity currents, Water Resour. Res. 42 (2006) W08424.

[5] N.J. Balmforth, R.V. Craster, A consistent thin-layer theory for Bingham plastics, J. Non-Newtonian Fluid Mech. 84 (1999) 65-81.

[6] C.M. Bender, S.A. Orszag, Advanced Mathematical Methods for Scientists and Engineers, Springer, New York, 1999.

[7] T.B. Benjamin, Gravity currents and related phenomena, J. Fluid Mech. 31 (1968) 209-248.

[8] G.W. Bluman, Invariant solutions for ordinary differential equations, SIAM J. Appl. Math. 50 (1990) 1706-1715.

[9] G.W. Bluman, S.C. Anco, Symmetry and Integration Methods for Differential Equations, Springer, New York, 2002.

[10] P.A. Blythe, Y. Kazakia, E. Varley, The interaction of large amplitude shallow-water waves with an ambient shear flows: Non-critical flows, J. Fluid Mech. 56 (1972) 241-255.

[11] R.I. Bowles, F.T. Smith, The standing hydraulic jump: Theory, computations and comparisons with experiments, J. Fluid Mech. 242 (1992) 145-168.

[12] B.J. Cantwell, Similarity transformations for the two-dimensional, unsteady, stream-function equation, J. Fluid Mech. 85 (1978) 257-271.

[13] G.F. Carrier, H.P. Greenspan, Water waves of finite amplitude on a sloping beach, J. Fluid Mech. 4 (1958) 97-109.

[14] H. Chanson, The Hydraulics of Open Channel Flow: An Introduction, 2nd edition, Elsevier-Butterworth-Heinemann, Amsterdam, 2004.

[15] P.A. Clarkson, T.J. Priestley, Shallow water wave systems, Stud. Appl. Math. 101 (1998) 389-432.

[16] R. Crocce, M. Griebel, M.A. Schweitzer, A parallel level-set approach for two-phase flow problems with surface tension in three space dimensions, Technical Report 157, Universität Bonn, Sonderforschungsbereich 611, 2004.

[17] S. Dartevelle, W.I. Rose, J. Stix, K. Kelfoun, J. Vallance, Numerical modeling of geophysical granular flows: 2. Computer simulations of plinian clouds and pyroclastic flows and surges, Geochem. Geophys. Geosyst. 5 (2004) 2003 GC000637.

[18] H.-S. Dou, N. Phan-Thien, B.C. Khoo, K.S. Yeo, R. Zheng, Simulation of front evolving liquid film down an inclined plate using level set method, Comput. Mech. 34 (2004) 271-281.

[19] L. Dressler, Similarity Solutions of Nonlinear Partial Differential Equations, Pitman, Boston, 1983.

[20] R.H. Dressler, Unsteady non-linear waves in sloping channels, Proc. R. Soc. Lond. Ser. A 247 (1958) 186-198.

[21] T.K. Fanneløp, G.D. Waldman, Dynamics of oil slicks, AIAA J. 10 (1972) 506-510.

[22] R. Fazio, A similarity approach to the numerical solution of free boundary problems, SIAM Rev. 40 (1998) 616-635.

[23] L.E. Fraenkel, On corner eddies in plane inviscid shear flows, J. Fluid Mech. 11 (1961) 400-406.

[24] N.C. Freeman, Simple waves on shear flows: Similarity solutions, J. Fluid Mech. 56 (1972) 257-263.

[25] J. Gratton, C. Vigo, Self-similar gravity currents with variable inflow revisited: Plane currents, J. Fluid Mech. 258 (1994) 77-104. 
[26] R.E. Grundy, J.W. Rottman, The approach to self-similarity of the solutions of the shallow-water equations representing gravity-current releases, J. Fluid Mech. 156 (1985) 39-53.

[27] R.E. Grundy, J.W. Rottman, Self-similar solutions of the shallow-water equations representing gravity currents with variable inflow, J. Fluid Mech. 169 (1986) 337-351.

[28] W.H. Hager, Forchheimer's and Schoklitsch's contributions to dambreak waves, in: W.H. Graf (Ed.), Philipp Forchheimer \& Armin Schoklitsch Memorial Symposium, IAHR Press, Graz, Austria, 1999, pp. 103-111.

[29] F.J. Higuera, The hydraulic jump in a viscous laminar flow, J. Fluid Mech. 274 (1994) 69-92.

[30] A.J. Hogg, D. Pritchard, The effects of hydraulic resistance on dam-break and other shallow inertial flows, J. Fluid Mech. 501 (2004) 179-212.

[31] H.G. Hornung, C. Willert, S. Turner, The flow field downstream of a hydraulic jump, J. Fluid Mech. 287 (1995) 299-316.

[32] D.P. Hoult, Oil spreading on the sea, Annu. Rev. Fluid Mech. 4 (1972) 341-368.

[33] H.E. Huppert, Gravity currents: A personal perspective, J. Fluid Mech. 554 (2006) 299-322.

[34] H.E. Huppert, The propagation of two-dimensional and axisymmetric viscous gravity currents over a rigid horizontal surface, J. Fluid Mech. 121 (1982) 43-58.

[35] H.E. Huppert, J.E. Simpson, The slumping of gravity currents, J. Fluid Mech. 99 (1980) 785-799.

[36] N.H. Ibragimov (Ed.), CRC Handbook of Lie Group Analysis of Differential Equations, CRC Press, Boca Raton, 1995.

[37] R.M. Iverson, The physics of debris flows, Rev. Geophys. 35 (1997) 245-296.

[38] C.W. Jones, On reducible non-linear differential equations occurring in mechanics, Proc. R. Soc. Lond. Ser. A 217 (1953) 327-343.

[39] J.B. Klemp, R. Rotunno, W.C. Skamarock, On the dynamics of gravity currents in a channel, J. Fluid Mech. 269 (1994) 169-198.

[40] M.J. Lighthill, G.B. Whitham, On kinematic waves. I. Flood movement in long rivers, Proc. R. Soc. Lond. Ser. A 229 (1955) 281-316.

[41] K.F. Liu, C.C. Mei, Approximate equations for the slow spreading of a thin sheet of Bingham plastic fluid, Phys. Fluids A 2 (1990) 30-36.

[42] M.S. Longuet-Higgins, A class of exact, time-dependent, free-surface flows, J. Fluid Mech. 55 (1972) 529-543.

[43] M.S. Longuet-Higgins, Self-similar, time-dependent flows with a free surface, J. Fluid Mech. 73 (1976) 603-620.

[44] B. Marino, L.P. Thomas, P.F. Linden, The front condition for gravity currents, J. Fluid Mech. 536 (2005) 49-78.

[45] T. Maxworthy, Gravity currents with variable inflow, J. Fluid Mech. 128 (1983) 247-257.

[46] J.N. McElwaine, Rotational flow in gravity current heads, Philos. Trans. R. Soc. Lond. Ser. A 363 (2005) 1603-1623.
[47] P.J. Montgomery, T.B. Moodie, Jump conditions for hyperbolic systems of forced conservation laws with an application to gravity currents, Stud. Appl. Math. 106 (2001) 367-392.

[48] T.G. Myers, Application of non-Newtonian models to thin film flow, Phys. Rev. E 72 (2005) 066302.

[49] A. Oron, S.H. Davis, S.G. Bankoff, Long-scale evolution of thin liquid films, Rev. Modern Phys. 69 (1997) 931-980.

[50] C.A. Perazzo, J. Gratton, Thin film of non-Newtonian fluid on an incline, Phys. Rev. E 67 (2003) 016307.

[51] M. Renardy, Well-posedness of the shallow-water equations in the presence of a front, Differential Integral Equations 11 (1998) 95-105.

[52] A. Ritter, Die Fortpflanzung der Wasserwellen, Zeit. Vereines Deutsch. Ing. 36 (33) (1892) 947-954.

[53] J.W. Rottman, J.E. Simpson, Gravity currents produced by instantaneous releases of a heavy fluid in a rectangular channel, J. Fluid Mech. 135 (1983) 95-110.

[54] A.C. Ruo, F. Chen, A similarity transformation for inviscid nonBoussinesq gravity currents, Acta Mech. 173 (2004) 33-40.

[55] P.L. Sachdev, Exact, self-similar, time-dependent free surface flows under gravity, J. Fluid Mech. 96 (1980) 797-802.

[56] B. Saint Venant, Théorie du mouvement non permanent des eaux, avec application aux crues des rivières et à l'introduction des marées dans leur lit, C. R. Acad. Sci. Paris Sér. I 173 (1871) 147-154. 237-240.

[57] S.B. Savage, K. Hutter, The motion of a finite mass of granular material down a rough incline, J. Fluid Mech. 199 (1989) 177-215.

[58] J.E. Simpson, Effects of a lower boundary on the head of a gravity current, J. Fluid Mech. 53 (1972) 759-768.

[59] J.E. Simpson, Gravity Currents in the Environment and the Laboratory, Ellis Horwood Limited, Chichester, 1987.

[60] J.J. Stoker, Water Waves, Interscience Publishers, New York, 1957.

[61] E.O. Tuck, L.W. Schwartz, A numerical and asymptotic study of some third-order ordinary differential equations relevant to draining and coating flows, SIAM Rev. 32 (1990) 453-469.

[62] T. van Kármán, The engineer grapples with nonlinear problems, Bull. Amer. Math. Soc. 46 (1940) 615-683.

[63] S. Watanabe, V. Putkaradze, T. Bohr, Integral methods for shallow freesurface flows with separation, J. Fluid Mech. 480 (2003) 233-265.

[64] S.J. Weinstein, K.J. Ruschak, Coating flows, Annu. Rev. Fluid Mech. 36 (2004) 29-53.

[65] G.B. Whitham, The effects of hydraulic resistance in the dam-break problem, Proc. R. Soc. Lond. Ser. A 227 (1954) 399-407.

[66] G.B. Whitham, Linear and Nonlinear Waves, John Wiley \& Sons, New York, 1974.

[67] J. Yu, J. Kevorkian, Nonlinear evolution of small disturbances into roll waves in an inclined open channel, J. Fluid Mech. 243 (1992) 575-594. 